\title{
Siegel families with application to class fields
}

\author{
Ja Kyung Koo \\ Department of Mathematical Sciences, KAIST, Daejeon 34141, \\ Republic of Korea (jkkoo@math.kaist.ac.kr)
}

\section{Dong Hwa Shin}

Department of Mathematics, Hankuk University of Foreign Studies, Yongin-si, Gyeonggi-do 17035, Republic of Korea (dhshin@hufs.ac.kr)

\author{
Dong Sung Yoon*广 \\ Department of Mathematical Sciences, KAIST, Daejeon 34141, \\ Republic of Korea
}

(MS received 24 March 2016; accepted 21 September 2016)

\begin{abstract}
We investigate certain families of meromorphic Siegel modular functions on which Galois groups act in a natural way. By using Shimura's reciprocity law we construct some algebraic numbers in the ray class fields of CM-fields in terms of special values of functions in these Siegel families.

Keywords: abelian varieties; class field theory; Shimura's reciprocity law; CM-fields; Siegel modular functions

2010 Mathematics subject classification: Primary 11F46

Secondary 11G15
\end{abstract}

\section{Introduction}

For a positive integer $N$ let $\mathfrak{F}_{N}$ be the field of meromorphic modular functions of level $N$ (defined on $\mathbb{H}=\{\tau \in \mathbb{C} \mid \operatorname{Im}(\tau)>0\}$ ) whose Fourier coefficients belong to the $N$ th cyclotomic field. As is well known, $\mathfrak{F}_{N}$ is a Galois extension of $\mathfrak{F}_{1}$ whose Galois group is isomorphic to $\mathrm{GL}_{2}(\mathbb{Z} / N \mathbb{Z}) /\left\{ \pm I_{2}\right\}$ (see $\left.[11, \S 6.1-6.2]\right)$. Now, let $N \geqslant 2$ and consider a set

$$
V_{N}=\left\{\boldsymbol{v} \in \mathbb{Q}^{2} \mid N \text { is the smallest positive integer for which } N \boldsymbol{v} \in \mathbb{Z}^{2}\right\}
$$

as the index set. We call a family $\left\{f_{\boldsymbol{v}}(\tau)\right\}_{\boldsymbol{v} \in V_{N}}$ of functions in $\mathfrak{F}_{N}$ a Fricke family of level $N$ if each $f_{\boldsymbol{v}}(\tau)$ depends only on $\pm \boldsymbol{v}\left(\bmod \mathbb{Z}^{2}\right)$ and satisfies

$$
f_{\boldsymbol{v}}(\tau)^{\alpha}=f_{\alpha^{\mathrm{T}} \boldsymbol{v}}(\tau) \quad\left(\alpha \in \mathrm{GL}_{2}(\mathbb{Z} / N \mathbb{Z}) /\left\{ \pm I_{2}\right\}\right),
$$

* Present address: Department of Mathematical Sciences, Ulsan National Institute of Science and Technology, Ulsan 44919, Republic of Korea (dsyoon@unist.ac.kr).

$\dagger$ Corresponding author.

(C) 2018 The Royal Society of Edinburgh 
where $\alpha^{\mathrm{T}}$ means the transpose of $\alpha$. For example, Siegel functions of one variable form such a Fricke family of level $N$ [8, ch. 2, proposition 1.3] (see also [4] or [6]).

Let $K$ be an imaginary quadratic field with the ring of integers $\mathcal{O}_{K}$, and let $\mathfrak{f}$ be a proper non-trivial ideal of $\mathcal{O}_{K}$. We denote by $\mathrm{Cl}(\mathfrak{f})$ and $K_{\mathfrak{f}}$ the ray class group modulo $\mathfrak{f}$ and its corresponding ray class field modulo $\mathfrak{f}$, respectively. If $\left\{f_{\boldsymbol{v}}(\tau)\right\}_{\boldsymbol{v}}$ is a Fricke family of level $N$ in which every $f_{\boldsymbol{v}}(\tau)$ is holomorphic on $\mathbb{H}$, then we can assign to each ray class $\mathcal{C} \in \mathrm{Cl}(\mathfrak{f})$ an algebraic number $f_{\mathfrak{f}}(\mathcal{C})$ as a special value of a function in $\left\{f_{\boldsymbol{v}}(\tau)\right\}_{\boldsymbol{v}}$. Furthermore, we attain by Shimura's reciprocity law that $f_{\mathfrak{f}}(\mathcal{C})$ belongs to $K_{\mathfrak{f}}$ and satisfies

$$
f_{\mathfrak{f}}(\mathcal{C})^{\sigma_{\mathfrak{f}}(\mathcal{D})}=f_{\mathfrak{f}}(\mathcal{C D}) \quad(\mathcal{D} \in \mathrm{Cl}(\mathfrak{f})),
$$

where $\sigma_{\mathfrak{f}}$ is the Artin reciprocity map for $\mathfrak{f}$ (see [8, ch. 11, theorem 1.1]).

In this paper we shall define a Siegel family $\left\{h_{M}(Z)\right\}_{M}$ of level $N$ consisting of meromorphic Siegel modular functions of (higher) genus $g$ and level $N$, which is a generalization of a Fricke family of level $N$ in the case when $g=1$ (definition 3.1). It turns out that every Siegel family of level $N$ is induced from a meromorphic Siegel modular function for the congruence subgroup $\Gamma^{1}(N)$ with rational Fourier coefficients (theorem 3.5).

Let $K$ be a CM-field and let $\mathfrak{f}=N \mathcal{O}_{K}$. Given a Siegel family $\left\{h_{M}(Z)\right\}_{M}$ of level $N$, we shall introduce a number $h_{\mathfrak{f}}(\mathcal{C})$ as a special value of a function in $\left\{h_{M}(Z)\right\}_{M}$ for each ray class $\mathcal{C} \in \mathrm{Cl}(\mathfrak{f})$ (definition 5.4). Under certain assumptions on $K$ (assumption 5.1) we shall prove that if $h_{\mathfrak{f}}(\mathcal{C})$ is finite, then it lies in the ray class field $K_{\mathfrak{f}}$ whose Galois conjugates are of the same form (theorem 7.2 and corollary 7.3). To this end, we assign a principally polarized abelian variety to each non-trivial ideal of $\mathcal{O}_{K}$, and apply Shimura's reciprocity law to $h_{\mathrm{f}}(\mathcal{C})$.

On the other hand, we note that there is a remarkable paper by Grant [2] in which he generalized a classical formula of Eisenstein and obtained classes of $S$-units by evaluating abelian functions at the intersections of divisors on the Jacobian of the curve $y^{2}=x^{5}+\frac{1}{4}$. We hope that our invariant $h_{\mathfrak{f}}(\mathcal{C})$ obtained from a Siegel family in theorem 4.3 will contribute further towards finding a higher-dimensional analogue of an elliptic unit.

\section{Actions on Siegel modular functions}

First, we shall describe the Galois group between fields of meromorphic Siegel modular functions in a concrete way.

Let $g$ be a positive integer and let

$$
\eta_{g}=\left[\begin{array}{cc}
O_{g} & -I_{g} \\
I_{g} & O_{g}
\end{array}\right]
$$

For every commutative ring $R$ with unity we define

$$
\begin{aligned}
\operatorname{GSp}_{2 g}(R) & =\left\{\alpha \in \mathrm{GL}_{2 g}(R) \mid \alpha^{\mathrm{T}} \eta_{g} \alpha=\nu(\alpha) \eta_{g} \text { with } \nu(\alpha) \in R^{\times}\right\}, \\
\operatorname{Sp}_{2 g}(R) & =\left\{\alpha \in \operatorname{GSp}_{2 g}(R) \mid \nu(\alpha)=1\right\} .
\end{aligned}
$$

Let

$$
G=\operatorname{GSp}_{2 g}(\mathbb{Q})
$$


and let $G_{\mathbb{A}}$ be the adelization of $G$, let $G_{0}$ be its non-Archimedean part and let $G_{\infty}$ be its Archimedean part. One can extend the multiplier map $\nu: G \rightarrow \mathbb{Q}^{\times}$ continuously to the map $\nu: G_{\mathbb{A}} \rightarrow \mathbb{Q}_{\mathbb{A}}^{\times}$and set

$$
G_{\infty+}=\left\{\alpha \in G_{\infty} \mid \nu(\alpha)>0\right\}, \quad G_{\mathbb{A}+}=G_{0} G_{\infty+}, \quad G_{+}=G \cap G_{\mathbb{A}+} .
$$

Furthermore, let

$$
\begin{aligned}
\Delta & =\left\{\left[\begin{array}{cc}
I_{g} & O_{g} \\
O_{g} & s I_{g}
\end{array}\right] \mid s \in \prod_{p} \mathbb{Z}_{p}^{\times}\right\}, \\
U_{1} & =\prod_{p} \operatorname{GSp}_{2 g}\left(\mathbb{Z}_{p}\right) \times G_{\infty+}, \\
U_{N} & =\left\{x \in U_{1} \mid x_{p} \equiv I_{2 g}\left(\bmod N \cdot M_{2 g}\left(\mathbb{Z}_{p}\right)\right) \text { for all rational primes } p\right\}
\end{aligned}
$$

for every positive integer $N$. Then we have

$$
U_{N} \unlhd U_{1} \leqslant G_{\mathbb{A}+} \quad \text { and } \quad G_{\mathbb{A}+}=U_{N} \Delta G_{+}
$$

(see [13, lemma 8.3(1)]).

Note that the group $G_{\infty+}$ acts on the Siegel upper half-space

$$
\mathbb{H}_{g}=\left\{Z \in M_{g}(\mathbb{C}) \mid Z^{\mathrm{T}}=Z, \operatorname{Im}(Z) \text { is positive definite }\right\}
$$

by

$$
\alpha(Z)=(A Z+B)(C Z+D)^{-1} \quad\left(\alpha \in G_{\infty+}, Z \in \mathbb{H}_{g}\right),
$$

where $A, B, C, D$ are $g \times g$ block matrices of $\alpha$. Let $\mathcal{F}_{N}$ be the field of meromorphic Siegel modular functions of genus $g$ for the congruence subgroup

$$
\Gamma(N)=\left\{\gamma \in \operatorname{Sp}_{2 g}(\mathbb{Z}) \mid \gamma \equiv I_{2 g}\left(\bmod N \cdot M_{2 g}(\mathbb{Z})\right)\right\}
$$

of the symplectic group $\operatorname{Sp}_{2 g}(\mathbb{Z})$ whose Fourier coefficients belong to the $N$ th cyclotomic field $\mathbb{Q}\left(\zeta_{N}\right)$ with $\zeta_{N}=\mathrm{e}^{2 \pi \mathrm{i} / N}$. That is, if $f \in \mathcal{F}_{N}$, then

$$
f(Z)=\frac{\sum_{h} c(h) e(\operatorname{tr}(h Z) / N)}{\sum_{h} d(h) e(\operatorname{tr}(h Z) / N)} \quad \text { for some } c(h), d(h) \in \mathbb{Q}\left(\zeta_{N}\right),
$$

where the denominator and numerator of $f$ are Siegel modular forms of the same weight, $h$ runs over all $g \times g$ positive semi-definite symmetric matrices over half integers with integral diagonal entries, and $e(w)=\mathrm{e}^{2 \pi \mathrm{i} w}$ for $w \in \mathbb{C}[5, \S 4$, theorem 1$]$. Let

$$
\mathcal{F}=\bigcup_{N=1}^{\infty} \mathcal{F}_{N} .
$$

Proposition 2.1. There exists a homomorphism $\tau: G_{\mathbb{A}+} \rightarrow \operatorname{Aut}(\mathcal{F})$ satisfying the following properties. Let

$$
f(Z)=\frac{\sum_{h} c(h) e(\operatorname{tr}(h Z) / N)}{\sum_{h} d(h) e(\operatorname{tr}(h Z) / N)} \in \mathcal{F}_{N} .
$$

(i) If $\alpha \in G_{+}=\{\alpha \in G \mid \nu(\alpha)>0\}$, then

$$
f^{\tau(\alpha)}=f \circ \alpha .
$$


(ii) If

$$
\beta=\left[\begin{array}{cc}
I_{g} & O_{g} \\
O_{g} & s I_{g}
\end{array}\right] \in \Delta
$$

and $t$ is a positive integer such that $t \equiv s_{p}\left(\bmod N \mathbb{Z}_{p}\right)$ for all rational primes $p$, then

$$
f^{\tau(\beta)}=\frac{\sum_{h} c(h)^{\sigma} e(\operatorname{tr}(h Z) / N)}{\sum_{h} d(h)^{\sigma} e(\operatorname{tr}(h Z) / N)},
$$

where $\sigma$ is the automorphism of $\mathbb{Q}\left(\zeta_{N}\right)$ given by $\zeta_{N}^{\sigma}=\zeta_{N}^{t}$.

(iii) For every positive integer $N$ we have

$$
\mathcal{F}_{N}=\left\{f \in \mathcal{F} \mid f^{\tau(x)}=f \text { for all } x \in U_{N}\right\} .
$$

(iv) We have $\operatorname{ker}(\tau)=\mathbb{Q}^{\times} G_{\infty+}$.

Proof. See [13, theorem 8.10].

Since

$$
U_{N}\left(\mathbb{Q}^{\times} G_{\infty+}\right) / \mathbb{Q}^{\times} G_{\infty+} \simeq U_{N} /\left(U_{N} \cap \mathbb{Q}^{\times} G_{\infty+}\right) \simeq \begin{cases}U_{1} / \pm G_{\infty+} & \text { if } N=1, \\ U_{N} / G_{\infty+} & \text { if } N>1,\end{cases}
$$

we see by proposition 2.1(iii) and (iv) that $\mathcal{F}_{N}$ is a Galois extension of $\mathcal{F}_{1}$ with

$$
\operatorname{Gal}\left(\mathcal{F}_{N} / \mathcal{F}_{1}\right) \simeq U_{1} / \pm U_{N} .
$$

Proposition 2.2. We have

$$
\operatorname{Gal}\left(\mathcal{F}_{N} / \mathcal{F}_{1}\right) \simeq \operatorname{GSp}_{2 g}(\mathbb{Z} / N \mathbb{Z}) /\left\{ \pm I_{2 g}\right\}
$$

Proof. Let $\alpha \in U_{1}$. Take a matrix $A$ in $M_{2 g}(\mathbb{Z})$ for which $A \equiv \alpha_{p}\left(\bmod N \cdot M_{2 g}\left(\mathbb{Z}_{p}\right)\right)$ for all rational primes $p$. Define a matrix $\psi(\alpha) \in M_{2 g}(\mathbb{Z} / N \mathbb{Z})$ by the image of $A$ under the natural reduction $M_{2 g}(\mathbb{Z}) \rightarrow M_{2 g}(\mathbb{Z} / N \mathbb{Z})$. Then, by the Chinese remainder theorem, $\psi(\alpha)$ is well defined and independent of the choice of $A$. Furthermore, let $t$ be an integer relatively prime to $N$ such that $t \equiv \nu\left(\alpha_{p}\right)\left(\bmod N \mathbb{Z}_{p}\right)$ for all rational primes $p$. We then derive that

$$
t \eta_{g} \equiv \nu\left(\alpha_{p}\right) \eta_{g} \equiv \alpha_{p}^{\mathrm{T}} \eta_{g} \alpha_{p} \equiv A^{\mathrm{T}} \eta_{g} A \equiv \psi(\alpha)^{\mathrm{T}} \eta_{g} \psi(\alpha) \quad\left(\bmod N \cdot M_{2 g}\left(\mathbb{Z}_{p}\right)\right)
$$

for all rational primes $p$, and hence $\psi(\alpha) \in \mathrm{GSp}_{2 g}(\mathbb{Z} / N \mathbb{Z})$. Thus, we obtain a group homomorphism

$$
\psi: U_{1} \rightarrow \mathrm{GSp}_{2 g}(\mathbb{Z} / N \mathbb{Z}) .
$$

Let $\beta \in \mathrm{GSp}_{2 g}(\mathbb{Z} / N \mathbb{Z})$ and take a preimage $B$ of $\beta$ under the natural reduction $M_{2 g}(\mathbb{Z}) \rightarrow M_{2 g}(\mathbb{Z} / N \mathbb{Z})$. Since $\nu(\beta) \in(\mathbb{Z} / N \mathbb{Z})^{\times}$and

$$
B^{\mathrm{T}} \eta_{g} B \equiv \beta^{\mathrm{T}} \eta_{g} \beta \equiv \nu(\beta) \eta_{g} \quad\left(\bmod N \cdot M_{2 g}(\mathbb{Z})\right),
$$

$B$ belongs to $\mathrm{GSp}_{2 g}\left(\mathbb{Z}_{p}\right)$ for every rational prime $p$ dividing $N$. Let $\alpha=\left(\alpha_{p}\right)_{p}$ be the element of $\prod_{p} \mathrm{GSp}_{2 g}\left(\mathbb{Z}_{p}\right)$ given by

$$
\alpha_{p}= \begin{cases}B & \text { if } p \mid N \\ I_{2 g} & \text { otherwise }\end{cases}
$$

We then see that $\alpha \in U_{1}$ and $\psi(\alpha)=\beta$. Thus, $\psi$ is surjective. 
Clearly, $U_{N}$ is contained in $\operatorname{ker}(\psi)$. Let $\gamma \in \operatorname{ker}(\psi)$. Since $\gamma_{p} \equiv I_{2 g}(\bmod N$. $\left.M_{2 g}\left(\mathbb{Z}_{p}\right)\right)$ for all rational primes $p$, we get $\gamma \in U_{N}$, and hence $\operatorname{ker}(\psi)=U_{N}$. Therefore, $\psi$ induces an isomorphism $U_{1} / U_{N} \simeq \operatorname{GSp}_{2 g}(\mathbb{Z} / N \mathbb{Z})$, from which we achieve, by $(2.1)$,

$$
\operatorname{Gal}\left(\mathcal{F}_{N} / \mathcal{F}_{1}\right) \simeq U_{1} / \pm U_{N} \simeq \operatorname{GSp}_{2 g}(\mathbb{Z} / N \mathbb{Z}) /\left\{ \pm I_{2 g}\right\}
$$

REMARK 2.3. We have the decomposition

$$
\operatorname{Gal}\left(\mathcal{F}_{N} / \mathcal{F}_{1}\right) \simeq \operatorname{GSp}_{2 g}(\mathbb{Z} / N \mathbb{Z}) /\left\{ \pm I_{2 g}\right\} \simeq G_{N} \cdot \operatorname{Sp}_{2 g}(\mathbb{Z} / N \mathbb{Z}) /\left\{ \pm I_{2 g}\right\}
$$

where

$$
G_{N}=\left\{\left[\begin{array}{cc}
I_{g} & O_{g} \\
O_{g} & \nu I_{g}
\end{array}\right] \mid \nu \in(\mathbb{Z} / N \mathbb{Z})^{\times}\right\} .
$$

By proposition 2.1 one can describe the action of $\mathrm{GSp}_{2 g}(\mathbb{Z} / N \mathbb{Z}) /\left\{ \pm I_{2 g}\right\}$ on $\mathcal{F}_{N}$ as follows.

Let

$$
f(Z)=\frac{\sum_{h} c(h) e(\operatorname{tr}(h Z) / N)}{\sum_{h} d(h) e(\operatorname{tr}(h Z) / N)} \in \mathcal{F}_{N}
$$

(i) An element

$$
\beta=\left[\begin{array}{cc}
I_{g} & O_{g} \\
O_{g} & \nu I_{g}
\end{array}\right]
$$

of $G_{N}$ acts on $f$ by

$$
f^{\beta}=\frac{\sum_{h} c(h)^{\sigma} e(\operatorname{tr}(h Z) / N)}{\sum_{h} d(h)^{\sigma} e(\operatorname{tr}(h Z) / N)},
$$

where $\sigma$ is the automorphism of $\mathbb{Q}\left(\zeta_{N}\right)$ satisfying $\zeta_{N}^{\sigma}=\zeta_{N}^{\nu}$.

(ii) An element $\gamma$ of $\operatorname{Sp}_{2 g}(\mathbb{Z} / N \mathbb{Z}) /\left\{ \pm I_{2 g}\right\}$ acts on $f$ by

$$
f^{\gamma}=f \circ \gamma^{\prime}
$$

where $\gamma^{\prime}$ is any preimage of $\gamma$ under the natural reduction

$$
\mathrm{Sp}_{2 g}(\mathbb{Z}) \rightarrow \operatorname{Sp}_{2 g}(\mathbb{Z} / N \mathbb{Z}) /\left\{ \pm I_{2 g}\right\}
$$

\section{Siegel families of level $N$}

By making use of the description of $\operatorname{Gal}\left(\mathcal{F}_{N} / \mathcal{F}_{1}\right)$ in $\S 2$ we shall introduce a generalization of a Fricke family in higher dimensional cases.

Let $N \geqslant 2$. For $\alpha \in M_{2 g}(\mathbb{Z})$ we denote by $\tilde{\alpha}$ its reduction modulo $N$. Define a set

$$
\mathcal{V}_{N}=\left\{(1 / N)\left[\begin{array}{l}
A^{\mathrm{T}} \\
B^{\mathrm{T}}
\end{array}\right] \mid \alpha=\left[\begin{array}{ll}
A & B \\
C & D
\end{array}\right] \in M_{2 g}(\mathbb{Z}) \text { such that } \tilde{\alpha} \in \operatorname{GSp}_{2 g}(\mathbb{Z} / N \mathbb{Z})\right\} .
$$

Let $\alpha, \beta$ be elements of $M_{2 g}(\mathbb{Z})$ satisfying $\alpha, \beta \in \mathrm{GSp}_{2 g}(\mathbb{Z} / N \mathbb{Z})$. If $M$ is an element of $\mathcal{V}_{N}$ induced from $\alpha$, then it is straightforward that $\beta^{\mathrm{T}} M$ is also an element of $\mathcal{V}_{N}$ given by the product $\alpha \beta$. 
Definition 3.1. We call a family $\left\{h_{M}(Z)\right\}_{M \in \mathcal{V}_{N}}$ a Siegel family of level $N$ if it satisfies the following:

(S1) each $h_{M}(Z)$ belongs to $\mathcal{F}_{N}$;

(S2) $h_{M}(Z)$ depends only on $\pm M\left(\bmod M_{2 g \times g}(\mathbb{Z})\right)$;

(S3) $h_{M}(Z)^{\sigma}=h_{\sigma^{\mathrm{T}} M}(Z)$ for all $\sigma \in \mathrm{GSp}_{2 g}(\mathbb{Z} / N \mathbb{Z}) /\left\{ \pm I_{2 g}\right\} \simeq \operatorname{Gal}\left(\mathcal{F}_{N} / \mathcal{F}_{1}\right)$.

By $\mathcal{S}_{N}$ we mean the set of such Siegel families of level $N$.

REMARK 3.2. Let $\left\{h_{M}(Z)\right\}_{M} \in \mathcal{S}_{N}$.

(i) The property (S3) yields a right action of the group $\operatorname{GSp}_{2 g}(\mathbb{Z} / N \mathbb{Z}) /\left\{ \pm I_{2 g}\right\}$ on $\left\{h_{M}(Z)\right\}_{M}$.

(ii) We let

$$
M=(1 / N)\left[\begin{array}{l}
A^{\mathrm{T}} \\
B^{\mathrm{T}}
\end{array}\right] \in \mathcal{V}_{N}
$$

and so there is a matrix

$$
\alpha=\left[\begin{array}{ll}
A & B \\
C & D
\end{array}\right] \in M_{2 g}(\mathbb{Z})
$$

such that $\tilde{\alpha} \in \mathrm{GSp}_{2 g}(\mathbb{Z} / N \mathbb{Z})$. Considering $\tilde{\alpha}$ as an element of $\mathrm{GSp}_{2 g}(\mathbb{Z} / N \mathbb{Z}) /$ $\left\{ \pm I_{2 g}\right\}$ we obtain

$$
h_{(1 / N)\left[\begin{array}{c}
I_{g} \\
O_{g}
\end{array}\right]}(Z)^{\tilde{\alpha}}=h_{(1 / N) \alpha^{\mathrm{T}}\left[\begin{array}{c}
I_{g} \\
O_{g}
\end{array}\right]}(Z)=h_{M}(Z) .
$$

Thus, the action of $\mathrm{GSp}_{2 g}(\mathbb{Z} / N \mathbb{Z}) /\left\{ \pm I_{2 g}\right\}$ on $\left\{h_{M}(Z)\right\}_{M}$ is transitive.

Let

$$
\Gamma^{1}(N)=\left\{\gamma \in \operatorname{Sp}_{2 g}(\mathbb{Z}) \mid \gamma \equiv\left[\begin{array}{cc}
I_{g} & O_{g} \\
* & I_{g}
\end{array}\right]\left(\bmod N \cdot M_{2 g}(\mathbb{Z})\right)\right\},
$$

and let $\mathcal{F}_{N}^{1}(\mathbb{Q})$ be the field of meromorphic Siegel modular functions for $\Gamma^{1}(N)$ with rational Fourier coefficients.

Lemma 3.3. If $\left\{h_{M}(Z)\right\}_{M} \in \mathcal{S}_{N}$, then

$$
h_{\left[\begin{array}{c}
(1 / N) I_{g} \\
O_{g}
\end{array}\right]}(Z) \in \mathcal{F}_{N}^{1}(\mathbb{Q}) .
$$

Proof. For any

$$
\gamma=\left[\begin{array}{ll}
A & B \\
C & D
\end{array}\right] \in \Gamma^{1}(N)
$$

we deduce by (S2) and (S3) that

$$
\begin{aligned}
h_{\left[\begin{array}{c}
(1 / N) I_{g} \\
O_{g}
\end{array}\right]}(\gamma(Z)) & =h_{\left[\begin{array}{c}
(1 / N) I_{g} \\
O_{g}
\end{array}\right]}(Z)^{\tilde{\gamma}}=h_{\gamma^{\mathrm{T}}\left[\begin{array}{c}
(1 / N) I_{g} \\
O_{g}
\end{array}\right]}(Z) \\
& =h_{(1 / N)\left[\begin{array}{c}
A^{\mathrm{T}} \\
B^{\mathrm{T}}
\end{array}\right]}(Z)=h_{\left[\begin{array}{c}
(1 / N) I_{g} \\
O_{g}
\end{array}\right]}(Z)
\end{aligned}
$$


because

$$
A \equiv I_{g}, \quad B \equiv O_{g} \quad\left(\bmod N \cdot M_{g}(\mathbb{Z})\right) .
$$

Thus, $h_{\left[\begin{array}{c}(1 / N) I_{g} \\ O_{g}\end{array}\right]}(Z)$ is modular for $\Gamma^{1}(N)$.

For every $\nu \in(\mathbb{Z} / N \mathbb{Z})^{\times}$we see by $(\mathrm{S} 2)$ and $(\mathrm{S} 3)$ that

$$
h_{\left[(1 / N) I_{g}\right.}(Z)^{\left[\begin{array}{cc}
I_{g} & O_{g} \\
O_{g} & \nu I_{g}
\end{array}\right]}=h_{\left[\begin{array}{cc}
I_{g} & O_{g} \\
O_{g} & \nu I_{g}
\end{array}\right]\left[\begin{array}{c}
(1 / N) I_{g} \\
O_{g}
\end{array}\right]}(Z)=h_{\left[\begin{array}{c}
(1 / N) I_{g} \\
O_{g}
\end{array}\right]}(Z),
$$
which implies that $h_{\left[\begin{array}{c}(1 / N) I_{g} \\ O_{g}\end{array}\right]}(Z)$ has rational Fourier coefficients. This proves the
lemma.

One can consider $\mathcal{S}_{N}$ as a field under the binary operations

$$
\begin{gathered}
\left\{h_{M}(Z)\right\}_{M}+\left\{k_{M}(Z)\right\}_{M}=\left\{\left(h_{M}+k_{M}\right)(Z)\right\}_{M}, \\
\left\{h_{M}(Z)\right\}_{M} \cdot\left\{k_{M}(Z)\right\}_{M}=\left\{\left(h_{M} k_{M}\right)(Z)\right\}_{M} .
\end{gathered}
$$

By lemma 3.3 we get the ring homomorphism

$$
\begin{aligned}
\phi_{N}: \mathcal{S}_{N} & \rightarrow \mathcal{F}_{N}^{1}(\mathbb{Q}) \\
\left\{h_{M}(Z)\right\}_{M} & \mapsto h_{\left[\begin{array}{c}
(1 / N) I_{g} \\
O_{g}
\end{array}\right]}(Z) .
\end{aligned}
$$

LEMmA 3.4. If $M \in \mathcal{V}_{N}$, then there exists

$$
\gamma=\left[\begin{array}{ll}
A & B \\
C & D
\end{array}\right] \in M_{2 g}(\mathbb{Z})
$$

such that $\tilde{\gamma} \in \mathrm{Sp}_{2 g}(\mathbb{Z} / N \mathbb{Z})$ and

$$
M=(1 / N)\left[\begin{array}{l}
A^{\mathrm{T}} \\
B^{\mathrm{T}}
\end{array}\right]
$$

Proof. Let

$$
\alpha=\left[\begin{array}{ll}
A & B \\
U & V
\end{array}\right] \in M_{2 g}(\mathbb{Z})
$$

such that $\tilde{\alpha} \in \mathrm{GSp}_{2 g}(\mathbb{Z} / N \mathbb{Z})$ and

$$
M=(1 / N)\left[\begin{array}{l}
A^{\mathrm{T}} \\
B^{\mathrm{T}}
\end{array}\right]
$$

In $M_{2 g}(\mathbb{Z} / N \mathbb{Z})$, decompose $\tilde{\alpha}$ as

$$
\tilde{\alpha}=\left[\begin{array}{cc}
I_{g} & O_{g} \\
O_{g} & \nu I_{g}
\end{array}\right]\left[\begin{array}{cc}
A & B \\
\nu^{-1} U & \nu^{-1} V
\end{array}\right] \quad \text { with } \nu=\nu(\tilde{\alpha}) \in(\mathbb{Z} / N \mathbb{Z})^{\times}
$$

so that

$$
\left[\begin{array}{cc}
A & B \\
\nu^{-1} U & \nu^{-1} V
\end{array}\right]
$$


belongs to $\mathrm{Sp}_{2 g}(\mathbb{Z} / N \mathbb{Z})$. Since the reduction $\mathrm{Sp}_{2 g}(\mathbb{Z}) \rightarrow \mathrm{Sp}_{2 g}(\mathbb{Z} / N \mathbb{Z})$ is surjective (see [10]), we can take $\gamma \in M_{2 g}(\mathbb{Z})$ satisfying

$$
\tilde{\gamma}=\left[\begin{array}{cc}
A & B \\
\nu^{-1} U & \nu^{-1} V
\end{array}\right] .
$$

Theorem 3.5. $\mathcal{S}_{N}$ and $\mathcal{F}_{N}^{1}(\mathbb{Q})$ are isomorphic via $\phi_{N}$.

Proof. Since $\mathcal{S}_{N}$ and $\mathcal{F}_{N}^{1}(\mathbb{Q})$ are fields, it suffices to show that $\phi_{N}$ is surjective.

Let $h(Z) \in \mathcal{F}_{N}^{1}(\mathbb{Q})$. For each $M \in \mathcal{V}_{N}$, take any

$$
\gamma=\left[\begin{array}{ll}
A & B \\
C & D
\end{array}\right] \in M_{2 g}(\mathbb{Z})
$$

such that $\tilde{\gamma} \in \operatorname{Sp}_{2 g}(\mathbb{Z} / N \mathbb{Z})$ and

$$
M=(1 / N)\left[\begin{array}{l}
A^{\mathrm{T}} \\
B^{\mathrm{T}}
\end{array}\right]
$$

by using lemma 3.4 . We set

$$
h_{M}(Z)=h(Z)^{\tilde{\gamma}} .
$$

We claim that $h_{M}(Z)$ is independent of the choice of $\gamma$. Indeed, if

$$
\gamma^{\prime}=\left[\begin{array}{cc}
A & B \\
C^{\prime} & D^{\prime}
\end{array}\right] \in M_{2 g}(\mathbb{Z})
$$

such that $\tilde{\gamma^{\prime}} \in \operatorname{Sp}_{2 g}(\mathbb{Z} / N \mathbb{Z})$, then we attain in $M_{2 g}(\mathbb{Z} / N \mathbb{Z})$ that

$$
\tilde{\gamma}^{\prime} \tilde{\gamma}^{-1}=\left[\begin{array}{cc}
A & B \\
C^{\prime} & D^{\prime}
\end{array}\right]\left[\begin{array}{cc}
D^{\mathrm{T}} & -B^{\mathrm{T}} \\
-C^{\mathrm{T}} & A^{\mathrm{T}}
\end{array}\right]=\left[\begin{array}{cc}
I_{g} & O_{g} \\
* & I_{g}
\end{array}\right]
$$

by the fact $\tilde{\gamma}, \tilde{\gamma^{\prime}} \in \operatorname{Sp}_{2 g}(\mathbb{Z} / N \mathbb{Z})$. Let $\delta$ be an element of $\operatorname{Sp}_{2 g}(\mathbb{Z})$ such that $\tilde{\delta}={\tilde{\gamma^{\prime}}}^{\prime} \tilde{\gamma}^{-1}$. We then achieve

$$
h(Z)^{\widetilde{\gamma^{\prime}}}=\left(h(Z)^{\widetilde{\gamma^{\prime}} \tilde{\gamma}^{-1}}\right)^{\tilde{\gamma}}=h(\delta(Z))^{\tilde{\gamma}}=h(Z)^{\tilde{\gamma}}
$$

because $h(Z)$ is modular for $\Gamma^{1}(N)$ and $\delta \in \Gamma^{1}(N)$.

Now, for any

$$
\sigma=\left[\begin{array}{ll}
P & Q \\
R & S
\end{array}\right] \in \mathrm{GSp}_{2 g}(\mathbb{Z} / N \mathbb{Z}) /\left\{ \pm I_{2 g}\right\}
$$

with $\nu=\nu(\sigma)$ we derive that

$$
\begin{aligned}
& h_{M}(Z)^{\sigma}=h(Z)^{\tilde{\gamma} \sigma} \\
& =h(Z)^{\left[\begin{array}{ll}
A & B \\
C & D
\end{array}\right]\left[\begin{array}{ll}
P & Q \\
R & S
\end{array}\right]} \\
& =h(Z) \underbrace{\left[\begin{array}{cc}
I_{g} & O_{g} \\
O_{g} & \nu I_{g}
\end{array}\right]}\left[\begin{array}{cc}
A P+B R & A Q+B S \\
\nu^{-1}(C P+D R) \nu^{-1}(C Q+D S)
\end{array}\right]
\end{aligned}
$$




$$
\begin{aligned}
= & h(Z)\left[\begin{array}{cc}
A P+B R & A Q+B S \\
\nu^{-1}(C P+D R) \nu^{-1}(C Q+D S)
\end{array}\right] \\
& \text { since } h(Z) \text { has rational Fourier coefficients } \\
= & h_{\left[\begin{array}{l}
(A P+B R)^{\mathrm{T}} \\
(A Q+B S)^{\mathrm{T}}
\end{array}\right]}(Z) \\
= & h_{\left[\begin{array}{ll}
P^{\mathrm{T}} & R^{\mathrm{T}} \\
Q^{\mathrm{T}} & S^{\mathrm{T}}
\end{array}\right]\left[\begin{array}{l}
A^{\mathrm{T}} \\
B^{\mathrm{T}}
\end{array}\right]}(Z) \\
= & h_{\sigma^{\mathrm{T}} M}(Z) .
\end{aligned}
$$

This shows that the family $\left\{h_{M}(Z)\right\}_{M}$ belongs to $\mathcal{S}_{N}$. Furthermore, since

$$
\phi_{N}\left(\left\{h_{M}(Z)\right\}_{M}\right)=h_{\left[\begin{array}{c}
(1 / N) I_{g} \\
O_{g}
\end{array}\right]}(Z)=h(Z)^{\left[\begin{array}{cc}
I_{g} & O_{g} \\
O_{g} & I_{g}
\end{array}\right]}=h(Z),
$$

$\phi$ is surjective as desired.

REMARK 3.6. (i) By proposition 2.2 and remark 2.3 we obtain

$$
\operatorname{Gal}\left(\mathcal{F}_{N} / \mathcal{F}_{N}^{1}(\mathbb{Q})\right) \simeq G_{N} \cdot\left\{\gamma \in \operatorname{Sp}_{2 g}(\mathbb{Z} / N \mathbb{Z}) /\left\{ \pm I_{2 g}\right\} \mid \gamma= \pm\left[\begin{array}{cc}
I_{g} & O_{g} \\
* & I_{g}
\end{array}\right]\right\}
$$

(ii) Let $\mathcal{F}_{1, N}(\mathbb{Q})$ be the field of meromorphic Siegel modular functions for

$$
\Gamma_{1}(N)=\left\{\gamma \in \operatorname{Sp}_{2 g}(\mathbb{Z}) \mid \gamma \equiv\left[\begin{array}{cc}
I_{g} & * \\
O_{g} & I_{g}
\end{array}\right]\left(\bmod N \cdot M_{2 g}(\mathbb{Z})\right)\right\}
$$

with rational Fourier coefficients. If we set

$$
\omega=\left[\begin{array}{cc}
(1 / \sqrt{N}) I_{g} & O_{g} \\
O_{g} & \sqrt{N} I_{g}
\end{array}\right]
$$

then we know that $\omega \in \mathrm{Sp}_{2 g}(\mathbb{R})$ and

$$
\omega\left[\begin{array}{cc}
A & B \\
C & D
\end{array}\right] \omega^{-1}=\left[\begin{array}{cc}
A & (1 / N) B \\
N C & D
\end{array}\right] \text { for all }\left[\begin{array}{cc}
A & B \\
C & D
\end{array}\right] \in \operatorname{Sp}_{2 g}(\mathbb{R}) .
$$

This implies

$$
\omega \Gamma^{1}(N) \omega^{-1}=\Gamma_{1}(N),
$$

and so $\mathcal{F}_{1, N}(\mathbb{Q})$ and $\mathcal{F}_{N}^{1}(\mathbb{Q})$ are isomorphic via

$$
\begin{aligned}
\mathcal{F}_{1, N}(\mathbb{Q}) & \rightarrow \mathcal{F}_{N}^{1}(\mathbb{Q}) \\
h(Z) & \mapsto(h \circ \omega)(Z)=h((1 / N) Z) .
\end{aligned}
$$

\section{An example of a Siegel family}

In this section, we shall give a concrete example of a Siegel family by means of theta constants.

Let $g$ be a positive integer. For

$$
\boldsymbol{v}=\left[\begin{array}{l}
\boldsymbol{v}_{u} \\
\boldsymbol{v}_{\ell}
\end{array}\right] \in \mathbb{Q}^{2 g}
$$


with $\boldsymbol{v}_{u}, \boldsymbol{v}_{\ell} \in \mathbb{Q}^{g}$, the theta constant $\theta_{\boldsymbol{v}}(Z)$ is given by

$$
\theta_{\boldsymbol{v}}(Z)=\sum_{\boldsymbol{n} \in \mathbb{Z}^{g}} e\left(\frac{1}{2}\left(\boldsymbol{n}+\boldsymbol{v}_{u}\right)^{\mathrm{T}} Z\left(\boldsymbol{n}+\boldsymbol{v}_{u}\right)+\left(\boldsymbol{n}+\boldsymbol{v}_{u}\right)^{\mathrm{T}} \boldsymbol{v}_{\ell}\right) \quad\left(Z \in \mathbb{H}_{g}\right) .
$$

It was shown by Igusa (see [3, theorem 2]) that $\theta_{\boldsymbol{v}}(Z)$ is identically zero if and only if every entry of the vector $\boldsymbol{v}$ is in $(1 / 2) \mathbb{Z}$ and $e\left(2 \boldsymbol{v}_{u}^{\mathrm{T}} \boldsymbol{v}_{\ell}\right)=-1$. Let

$$
S_{-}=\left\{\boldsymbol{a}=\left[\begin{array}{l}
\boldsymbol{a}_{u} \\
\boldsymbol{a}_{\ell}
\end{array}\right] \in\{0,1 / 2\}^{2 g} \mid e\left(2 \boldsymbol{a}_{u}^{\mathrm{T}} \boldsymbol{a}_{\ell}\right)=-1\right\} \quad \text { and } \quad S_{+}=\{0,1 / 2\}^{2 g} \backslash S_{-} .
$$

Now, let $\boldsymbol{v} \in \mathbb{Q}^{2 g}$ with exact denominator $N \geqslant 2$. We define

$$
\Theta_{\boldsymbol{v}}(Z)=2^{4 N} e\left(-2^{g} N\left(2^{g}-1\right)\left(2^{g}+1\right) \boldsymbol{v}_{u}^{\mathrm{T}} \boldsymbol{v}_{\ell}\right) \frac{\prod_{\boldsymbol{a} \in S_{-}} \theta_{\boldsymbol{a}-\boldsymbol{v}}(Z)^{4 N\left(2^{g}+1\right)}}{\prod_{\boldsymbol{b} \in S_{+}} \theta_{\boldsymbol{b}}(Z)^{4 N\left(2^{g}-1\right)}} \quad\left(Z \in \mathbb{H}_{g}\right)
$$

(see [7, definition 4.2]).

Proposition 4.1. The function $\Theta_{\boldsymbol{v}}(Z)$ depends only on $\pm \boldsymbol{v}\left(\bmod \mathbb{Z}^{2 g}\right)$. Moreover, it belongs to $\mathcal{F}_{N}$ and satisfies that

$$
\Theta_{\boldsymbol{v}}(Z)^{\sigma}=\Theta_{\sigma^{\mathrm{T}} \boldsymbol{v}}(Z)
$$

for every $\sigma \in \mathrm{GSp}_{2 g}(\mathbb{Z} / N \mathbb{Z}) /\left\{ \pm I_{2 g}\right\} \simeq \operatorname{Gal}\left(\mathcal{F}_{N} / \mathcal{F}_{1}\right)$.

Proof. See [7, lemma 4.4].

REMARK 4.2. One can readily verify that if $g \geqslant 2$, then $\Theta_{\boldsymbol{v}}(Z)$ is identically zero if and only if $N=2$.

Theorem 4.3. If $\boldsymbol{r} \in \mathbb{Q}^{g}$ with exact denominator $N \geqslant 3$, then $\left\{\Theta_{M(N \boldsymbol{r})}\right\}_{M \in \mathcal{V}_{N}}$ is a Siegel family of level $N$.

Proof. For any $\gamma \in \Gamma^{1}(N)$ we derive by proposition 4.1 that

$$
\Theta_{\left[\begin{array}{l}
\boldsymbol{r} \\
\mathbf{0}
\end{array}\right]}(\gamma(Z))=\Theta_{\left[\begin{array}{r}
\boldsymbol{r} \\
\mathbf{0}
\end{array}\right]}(Z)^{\tilde{\gamma}}=\Theta_{\gamma^{\mathrm{T}}\left[\begin{array}{l}
\boldsymbol{r} \\
\mathbf{0}
\end{array}\right]}(Z)=\Theta_{\left[\begin{array}{cc}
I_{g} & * \\
O_{g} & I_{g}
\end{array}\right]\left[\begin{array}{l}
\boldsymbol{r} \\
\mathbf{0}
\end{array}\right]}(Z)=\Theta_{\left[\begin{array}{c}
\boldsymbol{r} \\
\mathbf{0}
\end{array}\right]}(Z) .
$$

This shows that $\Theta_{\left[\begin{array}{r}\boldsymbol{r} \\ \mathbf{0}\end{array}\right]}(Z)$ is modular for $\Gamma^{1}(N)$. Furthermore, for any $\nu \in(\mathbb{Z} / N \mathbb{Z})^{\times}$, by proposition 4.1 we see that

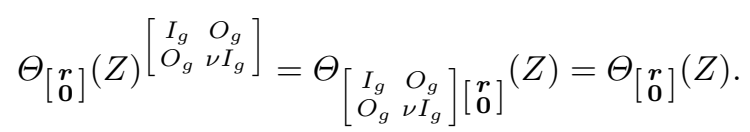

Thus, $\Theta_{\left[\begin{array}{l}\boldsymbol{r} \\ \mathbf{0}\end{array}\right]}(Z)$ has rational Fourier coefficients, and hence $\Theta_{\left[\begin{array}{l}\boldsymbol{r} \\ \mathbf{0}\end{array}\right]}(Z)$ belongs to $\mathcal{F}_{N}^{1}(\mathbb{Q})$.

For each $M \in \mathcal{V}_{N}$, we can take an element

$$
\gamma_{M}=\left[\begin{array}{ll}
A & B \\
C & D
\end{array}\right]
$$

of $M_{2 g}(\mathbb{Z})$ such that $\tilde{\gamma}_{M} \in \operatorname{Sp}_{2 g}(\mathbb{Z} / N \mathbb{Z})$ and

$$
M=(1 / N)\left[\begin{array}{l}
A^{\mathrm{T}} \\
B^{\mathrm{T}}
\end{array}\right]
$$




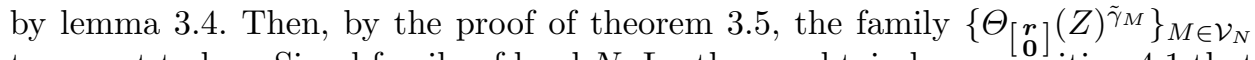
turns out to be a Siegel family of level $N$. Lastly, we obtain by proposition 4.1 that

$$
\Theta_{\left[\begin{array}{l}
\boldsymbol{r} \\
\mathbf{0}
\end{array}\right]}(Z)^{\tilde{\gamma}_{M}}=\Theta_{\gamma_{M}^{\mathrm{T}}\left[\begin{array}{l}
\boldsymbol{r} \\
\mathbf{0}
\end{array}\right]}(Z)=\Theta_{\left[\begin{array}{ll}
A^{\mathrm{T}} & C^{\mathrm{T}} \\
B^{\mathrm{T}} & D^{\mathrm{T}}
\end{array}\right]\left[\begin{array}{l}
\boldsymbol{r} \\
\mathbf{0}
\end{array}\right]}(Z)=\Theta_{\left[\begin{array}{l}
A^{\mathrm{T}} \\
B^{\mathrm{T}}
\end{array}\right] \boldsymbol{r}}(Z)=\Theta_{M(N \boldsymbol{r})}(Z) .
$$

This completes the proof.

\section{Special values associated with a Siegel family}

As an application of a Siegel family of level $N$ we shall construct a number associated with each ray class modulo $N$ of a CM-field.

Let $n$ be a positive integer, $K$ be a CM-field with $[K: \mathbb{Q}]=2 n$ and $\left\{\varphi_{1}, \ldots, \varphi_{n}\right\}$ be a set of embeddings of $K$ into $\mathbb{C}$ such that $\left(K,\left\{\varphi_{i}\right\}_{i=1}^{n}\right)$ is a CM-type. We fix a finite Galois extension $L$ of $\mathbb{Q}$ containing $K$, and set

$$
\begin{aligned}
S & =\left\{\sigma \in \operatorname{Gal}(L / \mathbb{Q})|\sigma|_{K}=\varphi_{i} \text { for some } i \in\{1,2, \ldots, n\}\right\}, \\
S^{*} & =\left\{\sigma^{-1} \mid \sigma \in S\right\}, \\
H^{*} & =\left\{\gamma \in \operatorname{Gal}(L / \mathbb{Q}) \mid \gamma S^{*}=S^{*}\right\} .
\end{aligned}
$$

Let $K^{*}$ be the subfield of $L$ corresponding to the subgroup $H^{*}$ of $\operatorname{Gal}(L / \mathbb{Q})$, and let $\left\{\psi_{1}, \ldots, \psi_{g}\right\}$ be the set of all embeddings of $K^{*}$ into $\mathbb{C}$ arising from the elements of $S^{*}$. Then we know that $\left(K^{*},\left\{\psi_{j}\right\}_{j=1}^{g}\right)$ is a primitive CM-type and

$$
K^{*}=\mathbb{Q}\left(\sum_{i=1}^{n} a^{\varphi_{i}} \mid a \in K\right)
$$

(see $\left[12, \S 8.3\right.$, proposition 28]). We call this CM-type $\left(K^{*},\left\{\psi_{j}\right\}_{j=1}^{g}\right)$ the reflex of $\left(K,\left\{\varphi_{i}\right\}_{i=1}^{n}\right)$. Using this CM-type we define an embedding

$$
\begin{aligned}
\Psi: K^{*} & \rightarrow \mathbb{C}^{g} \\
a & \mapsto\left[\begin{array}{c}
a^{\psi_{1}} \\
\vdots \\
a^{\psi_{g}}
\end{array}\right] .
\end{aligned}
$$

For each purely imaginary element $c$ of $K^{*}$ we associate an $\mathbb{R}$-bilinear form

$$
\begin{aligned}
E_{c}: \mathbb{C}^{g} \times \mathbb{C}^{g} & \rightarrow \mathbb{R} \\
(\boldsymbol{u}, \boldsymbol{v}) & \mapsto \sum_{j=1}^{g} c^{\psi_{j}}\left(u_{j} \bar{v}_{j}-\bar{u}_{j} v_{j}\right) \quad\left(\boldsymbol{u}=\left[\begin{array}{c}
u_{1} \\
\vdots \\
u_{g}
\end{array}\right], \boldsymbol{v}=\left[\begin{array}{c}
v_{1} \\
\vdots \\
v_{g}
\end{array}\right]\right) .
\end{aligned}
$$

Then, one can readily check that

$$
E_{c}(\Psi(a), \Psi(b))=\operatorname{Tr}_{K^{*} / \mathbb{Q}}(c a \bar{b}) \quad \text { for all } a, b \in K^{*}
$$

by using the fact $\overline{a^{\psi_{j}}}=\bar{a}^{\psi_{j}}$ for all $a \in K^{*}(1 \leqslant j \leqslant g)$. 
Assumption 5.1. In what follows we assume the following conditions.

(i) $\left(K^{*}\right)^{*}=K$.

(ii) There is a purely imaginary element $\xi$ of $K^{*}$ and a $\mathbb{Z}$-basis $\left\{\boldsymbol{a}_{1}, \ldots, \boldsymbol{a}_{2 g}\right\}$ of the lattice $\Psi\left(\mathcal{O}_{K^{*}}\right)$ in $\mathbb{C}^{g}$ for which

$$
\left[E_{\xi}\left(\boldsymbol{a}_{i}, \boldsymbol{a}_{j}\right)\right]_{1 \leqslant i, j \leqslant 2 g}=\left[\begin{array}{cc}
O_{g} & -I_{g} \\
I_{g} & O_{g}
\end{array}\right] .
$$

In this case, we say that the complex torus $\left(\mathbb{C}^{g} / \Psi\left(\mathcal{O}_{K^{*}}\right), E_{\xi}\right)$ is a principally polarized abelian variety with a symplectic basis $\left\{\boldsymbol{a}_{1}, \ldots, \boldsymbol{a}_{2 g}\right\}$. See $[12, \S 6.2]$.

(iii) $\mathfrak{f}=N \mathcal{O}_{K}$ for an integer $N \geqslant 2$.

REMARK 5.2. The assumption 5.1(i) is equivalent to saying that $\left(K,\left\{\varphi_{i}\right\}_{i=1}^{n}\right)$ is a primitive CM-type, namely, the abelian varieties of this CM-type are simple $[12$, $\S 8.2$, proposition 26].

By assumption 5.1(i) one can define a group homomorphism

$$
\begin{aligned}
\mathfrak{g}: K^{\times} & \rightarrow\left(K^{*}\right)^{\times} \\
d & \mapsto \prod_{i=1}^{n} d^{\varphi_{i}},
\end{aligned}
$$

and extend it continuously to the homomorphism $\mathfrak{g}: K_{\mathbb{A}}^{\times} \rightarrow\left(K^{*}\right)_{\mathbb{A}}^{\times}$of idele groups. It is also known that for each fractional ideal $\mathfrak{a}$ of $K$ there is a fractional ideal $\mathcal{G}(\mathfrak{a})$ of $K^{*}$ such that $[12, \S 8.3]$

$$
\mathcal{G}(\mathfrak{a}) \mathcal{O}_{L}=\prod_{i=1}^{n}\left(\mathfrak{a} \mathcal{O}_{L}\right)^{\varphi_{i}}
$$

Let $\mathcal{C}$ be a given ray class in $\mathrm{Cl}(\mathfrak{f})$. Take any integral ideal $\mathfrak{c}$ in $\mathcal{C}$, and let

$$
\mathcal{N}(\mathfrak{c})=\mathcal{N}_{K / \mathbb{Q}}(\mathfrak{c})=\left|\mathcal{O}_{K} / \mathfrak{c}\right|
$$

Lemma 5.3. $\left(\mathbb{C}^{g} / \Psi\left(\mathcal{G}(\mathfrak{c})^{-1}\right), E_{\xi \mathcal{N}(\mathfrak{c})}\right)$ is also a principally polarized abelian variety.

Proof. It follows from (5.1) that

$$
\begin{aligned}
E_{\xi \mathcal{N}(\mathfrak{c})}\left(\Psi\left(\mathcal{G}(\mathfrak{c})^{-1}\right), \Psi\left(\mathcal{G}(\mathfrak{c})^{-1}\right)\right) & =\operatorname{Tr}_{K^{*} / \mathbb{Q}}\left(\xi \mathcal{N}(\mathfrak{c}) \mathcal{G}(\mathfrak{c})^{-1} \overline{\mathcal{G}(\mathfrak{c})^{-1}}\right) \\
& =\operatorname{Tr}_{K^{*} / \mathbb{Q}}\left(\xi \mathcal{O}_{K^{*}}\right) \\
& =E_{\xi}\left(\Psi\left(\mathcal{O}_{K^{*}}\right), \Psi\left(\mathcal{O}_{K^{*}}\right)\right) \\
& \subseteq \mathbb{Z}
\end{aligned}
$$

because $E_{\xi}$ is a Riemann form on $\mathbb{C}^{g} / \Psi\left(\mathcal{O}_{K^{*}}\right)$. Thus, $E_{\xi \mathcal{N}(\mathfrak{c})}$ defines a Riemann form on $\mathbb{C}^{g} / \Psi\left(\mathcal{G}(\mathfrak{c})^{-1}\right)$.

Now, let $\left\{\boldsymbol{b}_{1}, \ldots, \boldsymbol{b}_{2 g}\right\}$ be a symplectic basis of the abelian variety $\left(\mathbb{C}^{g} / \Psi\left(\mathcal{G}(\mathfrak{c})^{-1}\right)\right.$, $\left.E_{\xi \mathcal{N}(\mathfrak{c})}\right)$ so that

$$
\Psi\left(\mathcal{G}(\mathfrak{c})^{-1}\right)=\sum_{j=1}^{2 g} \mathbb{Z} \boldsymbol{b}_{j} \quad \text { and } \quad\left[E_{\xi \mathcal{N}(\mathfrak{c})}\left(\boldsymbol{b}_{i}, \boldsymbol{b}_{j}\right)\right]_{1 \leqslant i, j \leqslant 2 g}=\left[\begin{array}{cc}
O_{g} & -\mathcal{E} \\
\mathcal{E} & O_{g}
\end{array}\right],
$$


where

$$
\mathcal{E}=\left[\begin{array}{ccc}
\varepsilon_{1} & \cdots & 0 \\
\vdots & \ddots & \vdots \\
0 & \cdots & \varepsilon_{g}
\end{array}\right]
$$

is a $g \times g$ diagonal matrix for some positive integers $\varepsilon_{1}, \ldots, \varepsilon_{g}$ satisfying $\varepsilon_{1}|\cdots| \varepsilon_{g}$. Furthermore, let $b_{1}, \ldots, b_{2 g}$ be elements of $\mathcal{G}(\mathfrak{c})^{-1}$ such that $\boldsymbol{b}_{j}=\Psi\left(b_{j}\right)(1 \leqslant j \leqslant$ $2 g$ ). Since $\mathcal{O}_{K^{*}} \subseteq \mathcal{G}(\mathfrak{c})^{-1}$, we have

$$
\left[\begin{array}{lll}
\boldsymbol{a}_{1} & \cdots & \boldsymbol{a}_{2 g}
\end{array}\right]=\left[\begin{array}{lll}
\boldsymbol{b}_{1} & \cdots & \boldsymbol{b}_{2 g}
\end{array}\right] \alpha \text { for some } \alpha \in M_{2 g}(\mathbb{Z}) \cap \mathrm{GL}_{2 g}(\mathbb{Q}),
$$

and hence

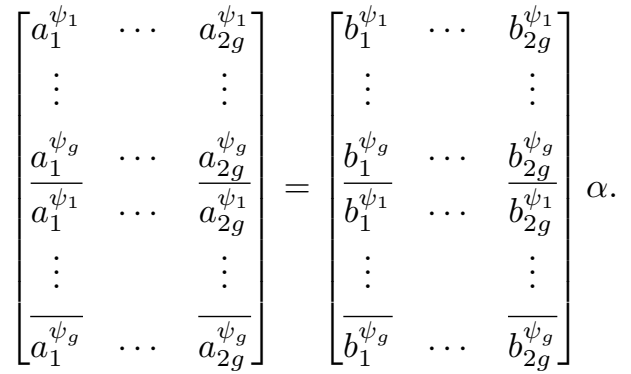

Taking determinant and squaring gives rise to the identity

$$
\Delta_{K * / \mathbb{Q}}\left(a_{1}, \ldots, a_{2 g}\right)=\Delta_{K * / \mathbb{Q}}\left(b_{1}, \ldots, b_{2 g}\right) \operatorname{det}(\alpha)^{2} .
$$

It then follows that

$$
\begin{aligned}
\operatorname{det}(\alpha)^{2} & =\frac{\left|\Delta_{K^{*} / \mathbb{Q}}\left(a_{1}, \ldots, a_{2 g}\right)\right|}{\left|\Delta_{K^{*} / \mathbb{Q}}\left(b_{1}, \ldots, b_{2 g}\right)\right|}=\frac{d_{K^{*} / \mathbb{Q}}\left(\mathcal{O}_{K^{*}}\right)}{d_{K^{*} / \mathbb{Q}}\left(\mathcal{G}(\mathfrak{c})^{-1}\right)} \\
& =\mathcal{N}_{K^{*} / \mathbb{Q}}(\mathcal{G}(\mathfrak{c}))^{2} \\
& =\mathcal{N}_{K^{*} / \mathbb{Q}}(\mathcal{G}(\mathfrak{c}) \overline{\mathcal{G}(\mathfrak{c})}) \\
& =\mathcal{N}(\mathfrak{c})^{2 g}
\end{aligned}
$$

where $d_{K^{*} / \mathbb{Q}}$ stands for the discriminant of a fractional ideal of $K^{*}[9$, ch. III, proposition 13]. Furthermore, we deduce by (5.2) that

$$
\begin{aligned}
\mathcal{N}(\mathfrak{c})\left[\begin{array}{cc}
O_{g} & -I_{g} \\
I_{g} & O_{g}
\end{array}\right] & =\left[\mathcal{N}(\mathfrak{c}) E_{\xi}\left(\boldsymbol{a}_{i}, \boldsymbol{a}_{j}\right)\right]_{1 \leqslant i, j \leqslant 2 g} \\
& =\left[E_{\xi \mathcal{N}(\mathfrak{c})}\left(\boldsymbol{a}_{i}, \boldsymbol{a}_{j}\right)\right]_{1 \leqslant i, j \leqslant 2 g} \\
& =\alpha^{\mathrm{T}}\left[E_{\xi \mathcal{N}(\mathfrak{c})}\left(\boldsymbol{b}_{i}, \boldsymbol{b}_{j}\right)\right]_{1 \leqslant i, j \leqslant 2 g} \alpha \\
& =\alpha^{\mathrm{T}}\left[\begin{array}{cc}
O_{g} & -\mathcal{E} \\
\mathcal{E} & O_{g}
\end{array}\right] \alpha
\end{aligned}
$$

By taking the determinant we get $\mathcal{N}(\mathfrak{c})^{2 g}=\operatorname{det}(\alpha)^{2}\left(\varepsilon_{1} \ldots \varepsilon_{g}\right)^{2}$, which, by (5.3), yields that $\varepsilon_{1}=\cdots=\varepsilon_{g}=1$, and so $\mathcal{E}=I_{g}$. Therefore, $\left(\mathbb{C}^{g} / \Psi\left(\mathcal{G}(\mathfrak{c})^{-1}\right), E_{\xi \mathcal{N}(\mathfrak{c})}\right)$ becomes a principally polarized abelian variety. 
As in the proof of lemma 5.3 we take a symplectic basis $\left\{\boldsymbol{b}_{1}, \ldots, \boldsymbol{b}_{2 g}\right\}$ of the principally polarized abelian variety $\left(\mathbb{C}^{g} / \Psi\left(\mathcal{G}(\mathfrak{c})^{-1}\right), E_{\xi \mathcal{N}(\mathfrak{c})}\right)$, and let $b_{1}, \ldots, b_{2 g}$ be elements of $\mathcal{G}(\mathfrak{c})^{-1}$ such that $\boldsymbol{b}_{j}=\Psi\left(b_{j}\right)(1 \leqslant j \leqslant 2 g)$. We then have

$$
\left[\begin{array}{lll}
\boldsymbol{a}_{1} & \cdots & \boldsymbol{a}_{2 g}
\end{array}\right]=\left[\begin{array}{lll}
\boldsymbol{b}_{1} & \cdots & \boldsymbol{b}_{2 g}
\end{array}\right] \alpha \quad \text { for some } \alpha=\left[\begin{array}{ll}
A & B \\
C & D
\end{array}\right] \in M_{2 g}(\mathbb{Z}) \cap \mathrm{GSp}_{2 g}(\mathbb{Q}) \text {. }
$$

Since $\nu(\alpha)=\mathcal{N}(\mathfrak{c})$ is relatively prime to $N$, the reduction $\tilde{\alpha}$ of $\alpha$ modulo $N$ belongs to $\mathrm{GSp}_{2 g}(\mathbb{Z} / N \mathbb{Z})$. Let $Z_{\mathfrak{c}}^{*}$ be the CM-point associated with the symplectic basis $\left\{\boldsymbol{b}_{1}, \ldots, \boldsymbol{b}_{2 g}\right\}$, namely

$$
Z_{\mathfrak{c}}^{*}=\left[\begin{array}{lll}
\boldsymbol{b}_{g+1} & \cdots & \boldsymbol{b}_{2 g}
\end{array}\right]^{-1}\left[\begin{array}{lll}
\boldsymbol{b}_{1} & \cdots & \boldsymbol{b}_{g}
\end{array}\right],
$$

which belongs to $\mathbb{H}_{g}[1$, proposition 8.1.1].

Definition 5.4. Let $\left\{h_{M}(Z)\right\}_{M} \in \mathcal{S}_{N}$. For a given ray class $\mathcal{C} \in \mathrm{Cl}(\mathfrak{f})$ we define

$$
h_{\mathfrak{f}}(\mathcal{C})=h_{(1 / N)\left[\begin{array}{l}
B \\
D
\end{array}\right]}\left(Z_{\mathfrak{c}}^{*}\right) .
$$

REMARK 5.5. Here, the index matrix

$$
(1 / N)\left[\begin{array}{l}
B \\
D
\end{array}\right]
$$

is obtained using the fact that

$$
\left(\left[\begin{array}{cc}
O_{g} & -I_{g} \\
I_{g} & O_{g}
\end{array}\right] \alpha\right)^{\mathrm{T}}=\left[\begin{array}{cc}
B^{\mathrm{T}} & D^{\mathrm{T}} \\
-A^{\mathrm{T}} & -C^{\mathrm{T}}
\end{array}\right]
$$

\section{Well-definedness of $h_{\mathfrak{f}}(\mathcal{C})$}

In this section we shall show that the value $h_{\mathfrak{f}}(\mathcal{C})$ given in definition 5.4 depends only on the ray class $\mathcal{C}$, and hence it is independent of the choice of a symplectic basis and an integral ideal in $\mathcal{C}$.

Proposition 6.1. The value $h_{\mathfrak{f}}(\mathcal{C})$ does not depend on the choice of a symplectic basis $\left\{\boldsymbol{b}_{1}, \ldots, \boldsymbol{b}_{2 g}\right\}$ of $\left(\mathbb{C}^{g} / \Psi\left(\mathcal{G}(\mathfrak{c})^{-1}\right), E_{\xi \mathcal{N}(\mathfrak{c})}\right)$.

Proof. Let $\left\{\widehat{\boldsymbol{b}}_{1}, \ldots, \widehat{\boldsymbol{b}}_{2 g}\right\}$ be another symplectic basis of $\left(\mathbb{C}^{g} / \Psi\left(\mathcal{G}(\mathfrak{c})^{-1}\right), E_{\xi \mathcal{N}(\mathfrak{c})}\right)$. Thus,

$$
\left[\begin{array}{lll}
\widehat{\boldsymbol{b}}_{1} & \cdots & \widehat{\boldsymbol{b}}_{2 g}
\end{array}\right]=\left[\begin{array}{lll}
\boldsymbol{b}_{1} & \cdots & \boldsymbol{b}_{2 g}
\end{array}\right] \beta \quad \text { for some } \beta=\left[\begin{array}{ll}
P & Q \\
R & S
\end{array}\right] \in \mathrm{GL}_{2 g}(\mathbb{Z})
$$

We then derive

$$
\begin{aligned}
{\left[\begin{array}{cc}
O_{g} & -I_{g} \\
I_{g} & O_{g}
\end{array}\right] } & =\left[E_{\xi \mathcal{N}(\mathfrak{c})}\left(\widehat{\boldsymbol{b}}_{i}, \widehat{\boldsymbol{b}}_{j}\right)\right]_{1 \leqslant i, j \leqslant 2 g} \\
& =\beta^{\mathrm{T}}\left[E_{\xi \mathcal{N}(\mathfrak{c})}\left(\boldsymbol{b}_{i}, \boldsymbol{b}_{j}\right)\right]_{1 \leqslant i, j \leqslant 2 g} \beta \\
& =\beta^{\mathrm{T}}\left[\begin{array}{cc}
O_{g} & -I_{g} \\
I_{g} & O_{g}
\end{array}\right] \beta,
\end{aligned}
$$


which shows that $\beta \in \operatorname{Sp}_{2 g}(\mathbb{Z})$. Since

$$
\left[\begin{array}{lll}
\boldsymbol{a}_{1} & \cdots & \boldsymbol{a}_{2 g}
\end{array}\right]=\left[\begin{array}{lll}
\boldsymbol{b}_{1} & \cdots & \boldsymbol{b}_{2 g}
\end{array}\right] \alpha=\left[\begin{array}{lll}
\widehat{\boldsymbol{b}}_{1} & \cdots & \widehat{\boldsymbol{b}}_{2 g}
\end{array}\right] \beta^{-1} \alpha
$$

by (5.4) and (6.1), the special value obtained by $\left\{\widehat{\boldsymbol{b}}_{1}, \ldots, \widehat{\boldsymbol{b}}_{2 g}\right\}$ is

$$
h_{(1 / N) \beta^{-1}\left[\begin{array}{l}
B \\
D
\end{array}\right]}\left(\hat{Z}_{\mathfrak{c}}^{*}\right)
$$

where $\hat{Z}_{\mathfrak{c}}^{*}$ is the CM-point corresponding to $\left\{\widehat{\boldsymbol{b}}_{1}, \ldots, \widehat{\boldsymbol{b}}_{2 g}\right\}$.

On the other hand, we attain that

$$
\begin{aligned}
& \hat{Z}_{\mathfrak{c}}^{*}=\left[\begin{array}{lll}
\widehat{\boldsymbol{b}}_{g+1} & \cdots & \widehat{\boldsymbol{b}}_{2 g}
\end{array}\right]^{-1}\left[\begin{array}{lll}
\widehat{\boldsymbol{b}}_{1} & \cdots & \widehat{\boldsymbol{b}}_{g}
\end{array}\right] \\
& =\left(\left[\begin{array}{lll}
\boldsymbol{b}_{1} & \cdots & \boldsymbol{b}_{g}
\end{array}\right] Q+\left[\begin{array}{lll}
\boldsymbol{b}_{g+1} & \cdots & \boldsymbol{b}_{2 g}
\end{array}\right] S\right)^{-1} \\
& \times\left(\left[\begin{array}{lll}
{\left[\boldsymbol{b}_{1}\right.} & \cdots & \boldsymbol{b}_{g}
\end{array}\right] P+\left[\begin{array}{lll}
\boldsymbol{b}_{g+1} & \cdots & \boldsymbol{b}_{2 g}
\end{array}\right] R\right) \text { by }(6.1)
\end{aligned}
$$

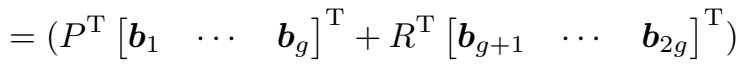

$$
\begin{aligned}
& \times\left(Q^{\mathrm{T}}\left[\begin{array}{lll}
\boldsymbol{b}_{1} & \cdots & \boldsymbol{b}_{g}
\end{array}\right]^{\mathrm{T}}+S^{\mathrm{T}}\left[\begin{array}{llll}
\boldsymbol{b}_{g+1} & \cdots & \boldsymbol{b}_{2 g}
\end{array}\right]^{\mathrm{T}}\right)^{-1}, \quad \text { since }\left(\hat{Z}_{\mathfrak{c}}^{*}\right)^{\mathrm{T}}=\hat{Z}_{\mathfrak{c}}^{*} \\
& =\left(P^{\mathrm{T}}\left(\left[\begin{array}{lll}
\boldsymbol{b}_{g+1} & \cdots & \boldsymbol{b}_{2 g}
\end{array}\right]^{-1}\left[\begin{array}{lll}
\boldsymbol{b}_{1} & \cdots & \boldsymbol{b}_{g}
\end{array}\right]\right)^{\mathrm{T}}+R^{\mathrm{T}}\right) \\
& \times\left(Q^{\mathrm{T}}\left(\left[\begin{array}{lll}
\boldsymbol{b}_{g+1} & \cdots & \boldsymbol{b}_{2 g}
\end{array}\right]^{-1}\left[\begin{array}{lll}
\boldsymbol{b}_{1} & \cdots & \boldsymbol{b}_{g}
\end{array}\right]\right)^{\mathrm{T}}+S^{\mathrm{T}}\right)^{-1} \\
& =\left(P^{\mathrm{T}}\left(Z_{\mathfrak{c}}^{*}\right)^{\mathrm{T}}+R^{\mathrm{T}}\right)\left(Q^{\mathrm{T}}\left(Z_{\mathfrak{c}}^{*}\right)^{\mathrm{T}}+S^{\mathrm{T}}\right)^{-1} \\
& =\left(P^{\mathrm{T}} Z_{\mathfrak{c}}^{*}+R^{\mathrm{T}}\right)\left(Q^{\mathrm{T}} Z_{\mathfrak{c}}^{*}+S^{\mathrm{T}}\right)^{-1} \quad \text { because }\left(Z_{\mathfrak{c}}^{*}\right)^{\mathrm{T}}=Z_{\mathfrak{c}}^{*} \\
& =\beta^{\mathrm{T}}\left(Z_{\mathrm{c}}^{*}\right) \text {. }
\end{aligned}
$$

Thus, we deduce that

$$
\begin{aligned}
h_{(1 / N) \beta^{-1}\left[\begin{array}{l}
B \\
D
\end{array}\right]}\left(\hat{Z}_{\mathfrak{c}}^{*}\right) & =h_{(1 / N) \beta^{-1}\left[\begin{array}{l}
B \\
D
\end{array}\right]}\left(\beta^{\mathrm{T}}\left(Z_{\mathfrak{c}}^{*}\right)\right) \quad \text { by }(6.2) \\
& =\left.\left(h_{(1 / N) \beta^{-1}\left[\begin{array}{l}
B \\
D
\end{array}\right]}(Z)\right)^{\beta^{\mathrm{T}}}\right|_{Z=Z_{\mathfrak{c}}^{*}} \\
& =h_{(1 / N)\left(\beta^{\mathrm{T}}\right)^{\mathrm{T}} \beta^{-1}\left[\begin{array}{l}
B \\
D
\end{array}\right]}\left(Z_{\mathfrak{c}}^{*}\right) \quad \text { by the property }\left(\text { S3) of }\left\{h_{M}(Z)\right\}_{M}\right. \\
& =h_{(1 / N)\left[\begin{array}{l}
B \\
D
\end{array}\right]}\left(Z_{\mathfrak{c}}^{*}\right) .
\end{aligned}
$$

This proves that the value $h_{\mathfrak{f}}(\mathcal{C})$ is independent of the choice of a symplectic basis of $\left(\mathbb{C}^{g} / \Psi\left(\mathcal{G}(\mathfrak{c})^{-1}\right), E_{\xi \mathcal{N}(\mathfrak{c})}\right)$.

REMARK 6.2. One can analogously readily show that $h_{\mathfrak{f}}(\mathcal{C})$ does not depend on the choice of a symplectic basis $\left\{\boldsymbol{a}_{1}, \ldots, \boldsymbol{a}_{2 g}\right\}$ of $\left(\mathbb{C}^{g} / \Psi\left(\mathcal{O}_{K}\right), E_{\xi}\right)$.

Proposition 6.3. $h_{\mathfrak{f}}(\mathcal{C})$ does not depend on the choice of an integral ideal $\mathfrak{c}$ in $\mathcal{C}$.

Proof. Let $\mathfrak{c}^{\prime}$ be another integral ideal in the class $\mathcal{C}$, and hence

$$
\mathfrak{c}^{\prime} \mathfrak{c}^{-1}=(1+a) \mathcal{O}_{K} \quad \text { for some } a \in \mathfrak{f a}^{-1}
$$


where $\mathfrak{a}$ is an integral ideal of $K$ relatively prime to $\mathfrak{f}$. Since $1 \in \mathfrak{c}^{-1}$ and $(1+a) \in$ $\mathfrak{c}^{\prime} \mathfrak{c}^{-1} \subseteq \mathfrak{c}^{-1}$, we get $a \in \mathfrak{c}^{-1}$. Thus, we derive that

$$
\begin{aligned}
a \mathfrak{a} \mathfrak{c} & \subseteq \mathfrak{f} \mathfrak{c} \cap \mathfrak{a} \quad \text { by the facts that } a \in \mathfrak{f a}^{-1} \text { and } a \in \mathfrak{c}^{-1} \\
& \subseteq \mathfrak{f} \cap \mathfrak{a} \\
& =\mathfrak{f a} \quad \text { because } \mathfrak{f} \text { and } \mathfrak{a} \text { are relatively prime, }
\end{aligned}
$$

from which it follows that $a \in \mathfrak{f c}^{-1}$. Using the fact that $\mathfrak{f}=N \mathcal{O}_{K}$ yields

$$
\begin{aligned}
\mathfrak{g}(1+a) & =\prod_{i=1}^{n}(1+a)^{\varphi_{i}} \in K^{*} \cap \prod_{i=1}^{n}\left(1+N\left(\mathfrak{c}^{-1} \mathcal{O}_{L}\right)^{\varphi_{i}}\right) \subseteq K^{*} \cap\left(1+N \mathcal{G}(\mathfrak{c})^{-1} \mathcal{O}_{L}\right) \\
& =1+N \mathcal{G}(\mathfrak{c})^{-1}
\end{aligned}
$$

Let

$$
b_{j}^{\prime}=\mathfrak{g}(1+a)^{-1} b_{j} \quad \text { and } \quad \boldsymbol{b}_{j}^{\prime}=\Psi\left(b_{j}^{\prime}\right) \quad(1 \leqslant j \leqslant 2 g) .
$$

We know that $\left\{\boldsymbol{b}_{1}^{\prime}, \ldots, \boldsymbol{b}_{2 g}^{\prime}\right\}$ is a $\mathbb{Z}$-basis of the lattice $\Psi\left(\mathcal{G}\left(\mathfrak{c}^{\prime}\right)^{-1}\right)$ in $\mathbb{C}^{g}$ and

$$
\boldsymbol{b}_{j}^{\prime}=T \boldsymbol{b}_{j} \quad \text { with } T=\left[\begin{array}{ccc}
\left(\mathfrak{g}(1+a)^{-1}\right)^{\psi_{1}} & \cdots & 0 \\
\vdots & \ddots & \vdots \\
0 & \cdots & \left(\mathfrak{g}(1+a)^{-1}\right)^{\psi_{g}}
\end{array}\right]
$$

Furthermore, we get that

$$
\begin{aligned}
& {\left[E_{\xi \mathcal{N}\left(\mathfrak{c}^{\prime}\right)}\left(\boldsymbol{b}_{i}^{\prime}, \boldsymbol{b}_{j}^{\prime}\right)\right]_{1 \leqslant i, j \leqslant 2 g}} \\
& =\left[\operatorname{Tr}_{K^{*} / \mathbb{Q}}\left(\xi \mathcal{N}\left(\mathfrak{c}^{\prime}\right) b_{i}^{\prime} \overline{b_{j}^{\prime}}\right)\right]_{1 \leqslant i, j \leqslant 2 g} \operatorname{by}(5.1) \\
& =\left[\operatorname{Tr}_{K^{*} / \mathbb{Q}}\left(\xi \mathcal{N}\left(\mathfrak{c}^{\prime}\right) \mathfrak{g}(1+a)^{-1} b_{i} \overline{\mathfrak{g}(1+a)^{-1} b_{j}}\right)\right]_{1 \leqslant i, j \leqslant 2 g} \quad \text { by }(6.5) \\
& =\left[\operatorname{Tr}_{K^{*} / \mathbb{Q}}\left(\xi \mathcal{N}\left(\mathfrak{c}^{\prime}\right) \mathrm{N}_{K / \mathbb{Q}}(1+a)^{-1} b_{i} \overline{b_{j}}\right)\right]_{1 \leqslant i, j \leqslant 2 g} \\
& =\left[\operatorname{Tr}_{K / \mathbb{Q}}\left(\xi \mathcal{N}(\mathfrak{c}) b_{i} \overline{b_{j}}\right)\right]_{1 \leqslant i, j \leqslant 2 g} \\
& \text { by (6.3) and the fact that } \mathrm{N}_{K / \mathbb{Q}}(1+a)>0 \\
& =\left[E_{\xi \mathcal{N}(\mathfrak{c})}\left(\boldsymbol{b}_{i}, \boldsymbol{b}_{j}\right)\right]_{1 \leqslant i, j \leqslant 2 g} \text { by }(5.1) \\
& =\left[\begin{array}{cc}
O_{g} & -I_{g} \\
I_{g} & O_{g}
\end{array}\right] \text {. }
\end{aligned}
$$

Thus, $\left\{\boldsymbol{b}_{1}^{\prime}, \ldots, \boldsymbol{b}_{2 g}^{\prime}\right\}$ is a symplectic basis of $\left(\mathbb{C}^{g} / \Psi\left(\mathcal{G}\left(\mathfrak{c}^{\prime}\right)^{-1}\right), E_{\xi \mathcal{N}\left(\mathfrak{c}^{\prime}\right)}\right)$, and its associated CM-point $Z_{\mathfrak{c}^{\prime}}^{*}$ is given by

$$
\begin{aligned}
Z_{\mathfrak{c}^{\prime}}^{*} & =\left[\begin{array}{lll}
\boldsymbol{b}_{g+1}^{\prime} & \cdots & \boldsymbol{b}_{2 g}^{\prime}
\end{array}\right]^{-1}\left[\begin{array}{lll}
\boldsymbol{b}_{1}^{\prime} & \cdots & \boldsymbol{b}_{g}^{\prime}
\end{array}\right] \\
& =\left[\begin{array}{llll}
T \boldsymbol{b}_{g+1} & \cdots & T \boldsymbol{b}_{2 g}
\end{array}\right]^{-1}\left[\begin{array}{llll}
T \boldsymbol{b}_{1} & \cdots & T \boldsymbol{b}_{g}
\end{array}\right] \text { by }(6.6) \\
& =Z_{\mathfrak{c}}^{*} .
\end{aligned}
$$

Let $\alpha=\left[a_{i j}\right], \alpha^{\prime}=\left[a_{i j}^{\prime}\right] \in M_{2 g}(\mathbb{Z})$ such that

$$
\left[\begin{array}{lll}
\boldsymbol{a}_{1} & \cdots & \boldsymbol{a}_{2 g}
\end{array}\right]=\left[\begin{array}{lll}
\boldsymbol{b}_{1} & \cdots & \boldsymbol{b}_{2 g}
\end{array}\right] \alpha=\left[\begin{array}{lll}
\boldsymbol{b}_{1}^{\prime} & \cdots & \boldsymbol{b}_{2 g}^{\prime}
\end{array}\right] \alpha^{\prime} .
$$


For each $1 \leqslant i \leqslant 2 g$ we obtain that

$$
\begin{aligned}
\sum_{j=1}^{2 g} a_{j i}^{\prime} b_{j} & =\mathfrak{g}(1+a) \sum_{j=1}^{2 g} a_{j i}^{\prime} b_{j}^{\prime} \quad \text { by }(6.5) \\
& =a_{i} \mathfrak{g}(1+a) \quad \text { by }(6.8) \\
& \in a_{i}\left(1+N \mathcal{G}(\mathfrak{c})^{-1}\right) \quad \text { by }(6.4) \\
& \subseteq a_{i}+N \mathcal{G}(\mathfrak{c})^{-1} \quad \text { because } a_{i} \in \mathcal{O}_{K} \\
& =\sum_{j=1}^{2 g} a_{j i} b_{j}+N \sum_{j=1}^{2 g} \mathbb{Z} b_{j} \quad \text { by }(6.8) .
\end{aligned}
$$

This yields $\alpha \equiv \alpha^{\prime}\left(\bmod N \cdot M_{2 g}(\mathbb{Z})\right)$, and hence

$$
(1 / N) \alpha \equiv(1 / N) \alpha^{\prime} \quad\left(\bmod M_{2 g}(\mathbb{Z})\right) .
$$

Now, the result follows from (6.7), (6.9) and the property (S2) of $\left\{h_{M}(Z)\right\}_{M}$.

\section{Galois actions on $h_{\mathfrak{f}}(\mathcal{C})$}

Finally, we shall show that if $h_{\mathfrak{f}}(\mathcal{C})$ is finite, then it lies in the ray class field $K_{\mathfrak{f}}$ and satisfies the natural transformation formula under the Artin reciprocity map for $\mathfrak{f}$.

Let $r: K^{*} \rightarrow M_{2 g}(\mathbb{Q})$ be the regular representation with respect to the ordered basis $\left\{a_{1}, \ldots, a_{2 g}\right\}$ of $K^{*}$ over $\mathbb{Q}$ given by

$$
a\left[\begin{array}{c}
a_{1} \\
\vdots \\
a_{2 g}
\end{array}\right]=r(a)\left[\begin{array}{c}
a_{1} \\
\vdots \\
a_{2 g}
\end{array}\right] \quad\left(a \in K^{*}\right) .
$$

Then it can be extended to the map $r:\left(K^{*}\right)_{\mathbb{A}} \rightarrow M_{2 g}\left(\mathbb{Q}_{\mathbb{A}}\right)$ of adele rings.

Lemma 7.1 (Shimura's reciprocity law). Let $f$ be an element of $\mathcal{F}$ that is finite at $Z_{\mathfrak{c}}^{*}$.

(i) The special value $f\left(Z_{\mathfrak{c}}^{*}\right)$ lies in $K_{\mathrm{ab}}$.

(ii) For every $s \in K_{\mathbb{A}}^{\times}$we have $r(\mathfrak{g}(s)) \in G_{\mathbb{A}+}$ and

$$
f\left(Z_{\mathfrak{c}}^{*}\right)^{[s, K]}=f^{\tau\left(r\left(\mathfrak{g}(s)^{-1}\right)\right)}\left(Z_{\mathfrak{c}}^{*}\right) .
$$

Proof. See [13, lemma 9.5 and theorem 9.6].

TheOREm 7.2. If $h_{\mathfrak{f}}(\mathcal{C})$ is finite, then it belongs to $K_{\mathfrak{f}}$. Furthermore, it satisfies

$$
h_{\mathfrak{f}}(\mathcal{C})^{\sigma_{\mathfrak{f}}(\mathcal{D})}=h_{\mathfrak{f}}(\mathcal{C D}) \quad \text { for every } \mathcal{D} \in \mathrm{Cl}(\mathfrak{f}),
$$

where $\sigma_{\mathfrak{f}}$ is the Artin reciprocity map for $\mathfrak{f}$.

Proof. Since $h_{\mathfrak{f}}(\mathcal{C})$ belongs to $K_{\mathrm{ab}}$ by lemma 7.1(i), there is a sufficiently large positive integer $M$ so that $N \mid M$ and $h_{\mathfrak{f}}(\mathcal{C}) \in K_{\mathfrak{m}}$ with $\mathfrak{m}=M \mathcal{O}_{K}$. Take an integral 
ideal $\mathfrak{d}$ in $\mathcal{D}$ relatively prime to $\mathfrak{m}$ by using the surjectivity of the natural map $\mathrm{Cl}(\mathfrak{m}) \rightarrow \mathrm{Cl}(\mathfrak{f})$. Let $\left\{\boldsymbol{d}_{1}, \ldots, \boldsymbol{d}_{2 g}\right\}$ be a symplectic basis of the principally polarized abelian variety $\left(\mathbb{C}^{g} / \Psi\left(\mathcal{G}(\mathfrak{c d})^{-1}\right), E_{\xi \mathcal{N}(\mathfrak{c} \mathfrak{d})}\right)$, and let $d_{1}, \ldots, d_{2 g}$ be elements of $\mathcal{G}(\mathfrak{c} \mathfrak{d})^{-1}$ such that $\boldsymbol{d}_{j}=\Psi\left(d_{j}\right)(1 \leqslant j \leqslant 2 g)$. Since $\mathcal{G}(\mathfrak{c})^{-1} \subseteq \mathcal{G}(\mathfrak{c} \mathfrak{d})^{-1}$, we get

$$
\left[\begin{array}{lll}
\boldsymbol{b}_{1} & \cdots & \boldsymbol{b}_{2 g}
\end{array}\right]=\left[\begin{array}{lll}
\boldsymbol{d}_{1} & \cdots & \boldsymbol{d}_{2 g}
\end{array}\right] \delta \quad \text { for some } \delta \in M_{2 g}(\mathbb{Z}) \cap \mathrm{GL}_{2 g}(\mathbb{Q}) .
$$

We then have that

$$
\begin{aligned}
{\left[\begin{array}{cc}
O_{g} & -I_{g} \\
I_{g} & O_{g}
\end{array}\right] } & =\left[E_{\xi \mathcal{N}(\mathfrak{c})}\left(\boldsymbol{b}_{i}, \boldsymbol{b}_{j}\right)\right]_{1 \leqslant i, j \leqslant 2 g} \\
& =\delta^{\mathrm{T}}\left[E_{\xi \mathcal{N}(\mathfrak{c})}\left(\boldsymbol{d}_{i}, \boldsymbol{d}_{j}\right)\right]_{1 \leqslant i, j \leqslant 2 g} \delta \quad \text { by }(7.2) \\
& =\delta^{\mathrm{T}}\left[\mathcal{N}(\mathfrak{c}) \mathcal{N}(\mathfrak{c} \mathfrak{d})^{-1} E_{\xi \mathcal{N}(\mathfrak{c} \mathfrak{d})}\left(\boldsymbol{d}_{i}, \boldsymbol{d}_{j}\right)\right]_{1 \leqslant i, j \leqslant 2 g} \delta \\
& =\mathcal{N}(\mathfrak{d})^{-1} \delta^{\mathrm{T}}\left[\begin{array}{cc}
O_{g} & -I_{g} \\
I_{g} & O_{g}
\end{array}\right] \delta .
\end{aligned}
$$

This claims that

$$
\delta \in M_{2 g}(\mathbb{Z}) \cap G_{+} \text {with } \nu(\delta)=\mathcal{N}(\mathfrak{d}) .
$$

Furthermore, if we let $Z_{\mathfrak{c o}}^{*}$ be the CM-point associated with $\left\{\boldsymbol{d}_{1}, \ldots, \boldsymbol{d}_{2 g}\right\}$, then we obtain

$$
Z_{\mathfrak{c d}}^{*}=\left(\delta^{-1}\right)^{\mathrm{T}}\left(Z_{\mathfrak{c}}^{*}\right)
$$

in a similar way to the argument in the proof of proposition 6.1 .

Let $s=\left(s_{p}\right)_{p}$ be an idele of $K$ such that

$$
\left.\begin{array}{rl}
s_{p}=1 & \text { if } p \mid M, \\
s_{p}\left(\mathcal{O}_{K}\right)_{p}=\mathfrak{d}_{p} & \text { if } p \nmid M .
\end{array}\right\}
$$

If we set $\tilde{\mathcal{D}}$ to be the ray class in $\mathrm{Cl}(\mathfrak{m})$ containing $\mathfrak{d}$, then by $(7.5)$ we attain

$$
\begin{aligned}
{\left.[s, K]\right|_{K_{\mathfrak{m}}} } & =\sigma_{\mathfrak{m}}(\tilde{\mathcal{D}}), \\
\mathfrak{g}(s)_{p}^{-1}\left(\mathcal{O}_{K^{*}}\right)_{p} & =\mathcal{G}(\mathfrak{d})_{p}^{-1} \quad \text { for all rational primes } p .
\end{aligned}
$$

It then follows from (7.1)-(7.7) that for every rational prime $p$, the entries of each of the vectors

$$
r\left(\mathfrak{g}(s)^{-1}\right)_{p}\left[\begin{array}{c}
b_{1} \\
\vdots \\
b_{2 g}
\end{array}\right] \quad \text { and } \quad\left(\delta^{-1}\right)^{\mathrm{T}}\left[\begin{array}{c}
b_{1} \\
\vdots \\
b_{2 g}
\end{array}\right]
$$

form a basis of $\mathcal{G}(\mathfrak{c} \mathfrak{d})_{p}^{-1}=\mathcal{G}(\mathfrak{c})^{-1} \mathcal{G}(\mathfrak{d})_{p}^{-1}$. So, there exists a matrix $u=\left(u_{p}\right)_{p} \in$ $\prod_{p} \mathrm{GL}_{2 g}\left(\mathbb{Z}_{p}\right)$ satisfying

$$
r\left(\mathfrak{g}(s)^{-1}\right)=u\left(\delta^{-1}\right)^{\mathrm{T}} .
$$

Since $\delta^{T}$ and

$$
\left[\begin{array}{cc}
I_{g} & O_{g} \\
O_{g} & \mathcal{N}(\delta) I_{g}
\end{array}\right]
$$


can be viewed as elements of $\operatorname{GSp}_{2 g}(Z / M \mathbb{Z})$ by (7.3), there exists a matrix $\gamma \in$ $\mathrm{Sp}_{2 g}(\mathbb{Z})$ such that

$$
\delta^{\mathrm{T}} \equiv\left[\begin{array}{cc}
I_{g} & O_{g} \\
O_{g} & \mathcal{N}(\delta) I_{g}
\end{array}\right] \gamma \quad\left(\bmod M \cdot M_{2 g}(\mathbb{Z})\right)
$$

owing to the surjectivity of the reduction $\mathrm{Sp}_{2 g}(\mathbb{Z}) \rightarrow \mathrm{Sp}_{2 g}(\mathbb{Z} / M \mathbb{Z})$. Since

$$
r\left(\mathfrak{g}(s)^{-1}\right)_{p}=I_{2 g} \quad \text { for all } p \mid M
$$

by (7.5), we get $u_{p}=\delta^{\mathrm{T}}$ for all $p \mid M$ by (7.8). Hence, we deduce using (7.9) that

$$
u_{p} \gamma^{-1} \equiv\left[\begin{array}{cc}
I_{g} & O_{g} \\
O_{g} & \mathcal{N}(\delta) I_{g}
\end{array}\right] \quad\left(\bmod M \cdot M_{2 g}\left(\mathbb{Z}_{p}\right)\right) \quad \text { for all rational primes } p .
$$

On the other hand, we have by (5.4) and (7.2) that

$$
\begin{aligned}
{\left[\begin{array}{lll}
\boldsymbol{a}_{1} & \cdots & \boldsymbol{a}_{2 g}
\end{array}\right] } & =\left[\begin{array}{lll}
\boldsymbol{b}_{1} & \cdots & \boldsymbol{b}_{2 g}
\end{array}\right] \alpha \\
& =\left(\left[\begin{array}{lll}
\boldsymbol{b}_{1} & \cdots & \boldsymbol{b}_{2 g}
\end{array}\right] \delta^{-1}\right)(\delta \alpha) \\
& =\left[\begin{array}{lll}
\boldsymbol{d}_{1} & \cdots & \boldsymbol{d}_{2 g}
\end{array}\right](\delta \alpha) .
\end{aligned}
$$

Letting

$$
\alpha=\left[\begin{array}{ll}
A & B \\
C & D
\end{array}\right]
$$

we induce the following:

$$
\begin{aligned}
& h_{\mathfrak{f}}(\mathcal{C})^{\sigma_{\mathfrak{m}}(\tilde{\mathcal{D}})}=h_{\mathfrak{f}}(\mathcal{C})^{[s, K]} \quad \text { by }(7.6) \\
& =h_{(1 / N)\left[\begin{array}{l}
B \\
D
\end{array}\right]}\left(Z_{\mathfrak{c}}^{*}\right)^{[s, K]} \text { by definition } 5.4 \\
& =\left.h_{(1 / N)\left[\begin{array}{l}
B \\
D
\end{array}\right]}(Z)^{\tau\left(r\left(\mathfrak{g}(s)^{-1}\right)\right)}\right|_{Z=Z_{\mathrm{c}}^{*}} \text { by lemma } 7.1(\mathrm{ii}) \\
& =\left.h_{(1 / N)\left[\begin{array}{l}
B \\
D
\end{array}\right]}(Z)^{\tau\left(u\left(\delta^{-1}\right)^{\mathrm{T}}\right)}\right|_{Z=Z_{\mathrm{c}}^{*}} \text { by }(7.8) \\
& =\left.h_{(1 / N)\left[\begin{array}{l}
B \\
D
\end{array}\right]}(Z)^{\tau\left(u \gamma^{-1}\right) \tau(\gamma) \tau\left(\left(\delta^{-1}\right)^{\mathrm{T}}\right)}\right|_{Z=Z_{c}^{*}}
\end{aligned}
$$

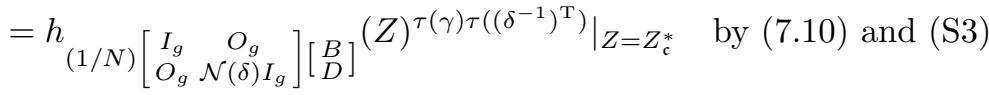

$$
\begin{aligned}
& =\left.h_{(1 / N) \gamma^{\mathrm{T}}}\left[\begin{array}{cc}
I_{g} & O_{g} \\
O_{g} & \mathcal{N}(\delta) I_{g}
\end{array}\right]\left[\begin{array}{l}
B \\
D
\end{array}\right](Z)^{\tau\left(\left(\delta^{-1}\right)^{\mathrm{T}}\right)}\right|_{Z=Z_{\mathrm{c}}^{*}} \quad \text { by }(\mathrm{S} 3) \\
& =\left.h_{(1 / N) \delta\left[\begin{array}{l}
B \\
D
\end{array}\right]}(Z)^{\tau\left(\left(\delta^{-1}\right)^{\mathrm{T}}\right)}\right|_{Z=Z_{\text {c }}^{*}} \text { by (7.9) and (S2) } \\
& =h_{(1 / N) \delta\left[\begin{array}{l}
B \\
D
\end{array}\right]}\left(\left(\delta^{-1}\right)^{\mathrm{T}}\left(Z_{\mathfrak{c}}^{*}\right)\right) \quad \text { due to the fact that } \delta \in G_{+} \text {and by (A1) } \\
& =h_{\mathfrak{f}}(\mathcal{C D}) \text { by (7.4), (7.11) and definition 5.4. }
\end{aligned}
$$

In particular, suppose that $\mathfrak{d}=d \mathcal{O}_{K}$ for some $d \in \mathcal{O}_{K}$ such that $d \equiv 1(\bmod \mathfrak{f})$. Then $\mathcal{D}$ is the identity class of $\mathrm{Cl}(\mathfrak{f})$, and so the above observation implies that $\sigma_{\mathfrak{m}}(\tilde{\mathcal{D}})$ leaves $h_{\mathfrak{f}}(\mathcal{C})$ fixed. Therefore, we conclude that $h_{\mathfrak{f}}(\mathcal{C})$ lies in $K_{\mathfrak{f}}$. 
Corollary 7.3. Let $H$ be a subgroup of $\mathrm{Cl}(\mathfrak{f})$ defined by

$H=\langle\mathcal{D} \in \mathrm{Cl}(\mathfrak{f})| \mathcal{D}$ contains an integral ideal $\mathfrak{d}$ of $K$ for which $\mathcal{G}(\mathfrak{d})=\mathfrak{g}(d) \mathcal{O}_{K^{*}}$

for some $d \in \mathcal{O}_{K}$ such that $\left.\mathfrak{g}(d) \equiv 1\left(\bmod N \mathcal{O}_{K^{*}}\right)\right\rangle$,

and let $K_{\mathfrak{f}}^{H}$ be the fixed field of $H$. If $h_{\mathfrak{f}}(\mathcal{C})$ is finite, then it belongs to $K_{\mathfrak{f}}^{H}$.

Proof. Let $\mathcal{C}_{0}$ be the identity class of $\mathrm{Cl}(\mathfrak{f})$. Since $h_{\mathfrak{f}}\left(\mathcal{C}_{0}\right) \in K_{\mathfrak{f}}$ by theorem 7.2 , $K\left(h_{\mathfrak{f}}\left(\mathcal{C}_{0}\right)\right)$ is a Galois extension of $K$ as a subfield of $K_{\mathfrak{f}}$. Furthermore, since

$$
h_{\mathfrak{f}}\left(\mathcal{C}_{0}\right)^{\sigma_{\mathfrak{f}}(\mathcal{C})}=h_{\mathfrak{f}}\left(\mathcal{C}_{0} \mathcal{C}\right)=h_{\mathfrak{f}}(\mathcal{C})
$$

by theorem $7.2, K\left(h_{\mathfrak{f}}\left(\mathcal{C}_{0}\right)\right)$ contains $h_{\mathfrak{f}}(\mathcal{C})$. Thus, it suffices to show that $h_{\mathfrak{f}}\left(\mathcal{C}_{0}\right)$ belongs to $K_{\mathrm{f}}^{H}$.

To this end, let $\mathcal{D}$ be an element of $\mathrm{Cl}(\mathfrak{f})$ containing an integral ideal $\mathfrak{d}$ of $K$ for which

$$
\mathcal{G}(\mathfrak{d})=\mathfrak{g}(d) \mathcal{O}_{K^{*}} \quad \text { for some } d \in \mathcal{O}_{K} \text { such that } \mathfrak{g}(d) \equiv 1 \quad\left(\bmod N \mathcal{O}_{K^{*}}\right) .
$$

Now that

$$
\left(\mathbb{C}^{g} / \Psi\left(\mathcal{G}(\mathfrak{d})^{-1}\right), E_{\xi \mathcal{N}(\mathfrak{d})}\right)=\left(\mathbb{C}^{g} / \Psi\left(\mathfrak{g}(d)^{-1} \mathcal{O}_{K^{*}}\right), E_{\xi \mathcal{N}\left(d \mathcal{O}_{K}\right)}\right)
$$

we obtain

$$
h_{\mathfrak{f}}\left(\mathcal{C}_{0}\right)^{\sigma_{\mathfrak{f}}(\mathcal{D})}=h_{\mathfrak{f}}(\mathcal{D})=h_{\mathfrak{f}}\left(\left[d \mathcal{O}_{K}\right]\right),
$$

where $[\mathfrak{a}]$ is the ray class containing $\mathfrak{a}$ for a fractional ideal $\mathfrak{a}$ of $K$. Moreover, since $\mathfrak{g}(d) \equiv 1\left(\bmod N \mathcal{O}_{K^{*}}\right)$, we obtain

$$
h_{\mathfrak{f}}\left(\left[d \mathcal{O}_{K}\right]\right)=h_{\mathfrak{f}}\left(\left[\mathcal{O}_{K}\right]\right)=h_{\mathfrak{f}}\left(\mathcal{C}_{0}\right)
$$

analogously to the proof of proposition 6.3 . This proves that $h_{\mathfrak{f}}\left(\mathcal{C}_{0}\right)$ belongs to $K_{\mathfrak{f}}^{H}$.

\section{Acknowledgements}

We thank the referee for valuable comments, which improved on an earlier version of the manuscript. D.H.S. was supported by the Hankuk University of Foreign Studies Research Fund of 2016.

\section{References}

1 C. Birkenhake and H. Lange. Complex abelian varieties. Grundlehren der mathematischen Wissenschaften, vol. 302 (Springer, 2004).

2 D. Grant. A generalization of a formula of Eisenstein. Proc. Lond. Math. Soc. 62 (1991), 121-132.

3 J.-I. Igusa. On the graded ring of theta-constants. II. Am. J. Math. 88 (1966), 221-236.

4 H. Y. Jung, J. K. Koo and D. H. Shin. On some Fricke families and application to the Lang-Schertz conjecture. Proc. R. Soc. Edinb. A 146 (2016), 723-740.

$5 \quad H$. Klingen. Introductory lectures on Siegel modular forms. Cambridge Studies in Advanced Mathematics, vol. 20 (Cambridge University Press, 1990).

6 J. K. Koo and D. S. Yoon. Generators of the ring of weakly holomorphic modular functions for $\Gamma_{1}(N)$. Ramanujan J. 42 (2017), 583-599. 
7 J. K. Koo, G. Robert, D. H. Shin and D. S. Yoon. On Siegel invariants of certain CM-fields. Preprint, 2015. (Available at http://arxiv.org/abs/1508.05602.)

8 D. Kubert and S. Lang. Modular units. Grundlehren der mathematischen Wissenschaften, vol. 244 (Spinger, 1981).

9 S. Lang. Algebraic number theory, 2nd edn. Graduate Texts in Mathematics, vol. 110 (Springer, 1986).

10 A. S. Rapinchuk. Strong approximation for algebraic groups. In Thin groups and superstrong approximation. MSRI Publications, vol. 61, pp. 269-298 (Cambridge University Press, 2014).

11 G. Shimura. Introduction to the arithmetic theory of automorphic functions. Publications of the Mathematical Society of Japan, vol. 11 (Princeton University Press, 1971).

12 G. Shimura. Abelian varieties with complex multiplication and modular functions (Princeton University Press, 1998).

13 G. Shimura. Arithmeticity in the theory of automorphic forms. Mathematical Surveys and Monographs, vol. 82 (Providence, RI: American Mathematical Society, 2000). 